\title{
ANNUAL REPORT1991
}

NISTIR 4759

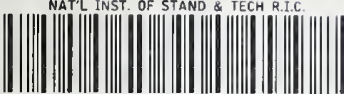

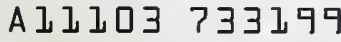

NIST

PUBLICATIONS
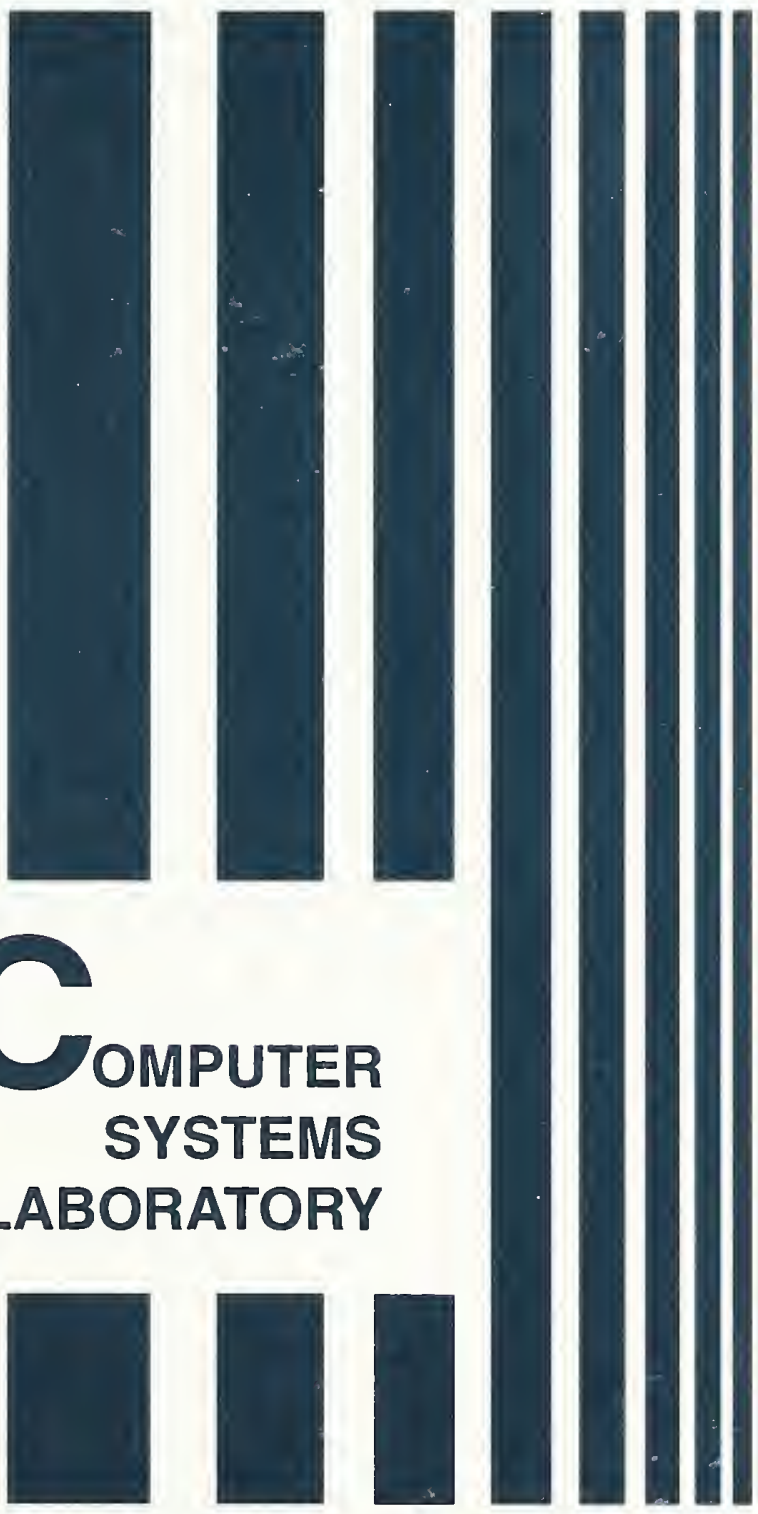

QC

100

.056

\#4759
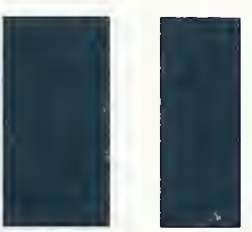

OMPUTER SYSTEMS

LABORATORY

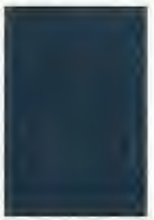

.

1991

C. 2

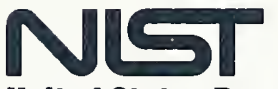

United States Department of Commerce

Technology Administration

National Institute of Standards and Technology 


\section{ANNUAL REPORT 1991}

NISTIR 4759

December 1991

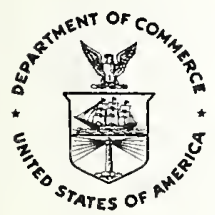

U.S. Department

of Commerce

Robert A. Mosbacher, Secretary

Technology Administration

Robert M. White,

Under Secretary

for Technology

National Institute of Standards and

Technology

John W. Lyons,

Director

Computer Systems

Laboratory

James H. Burrows,

Director

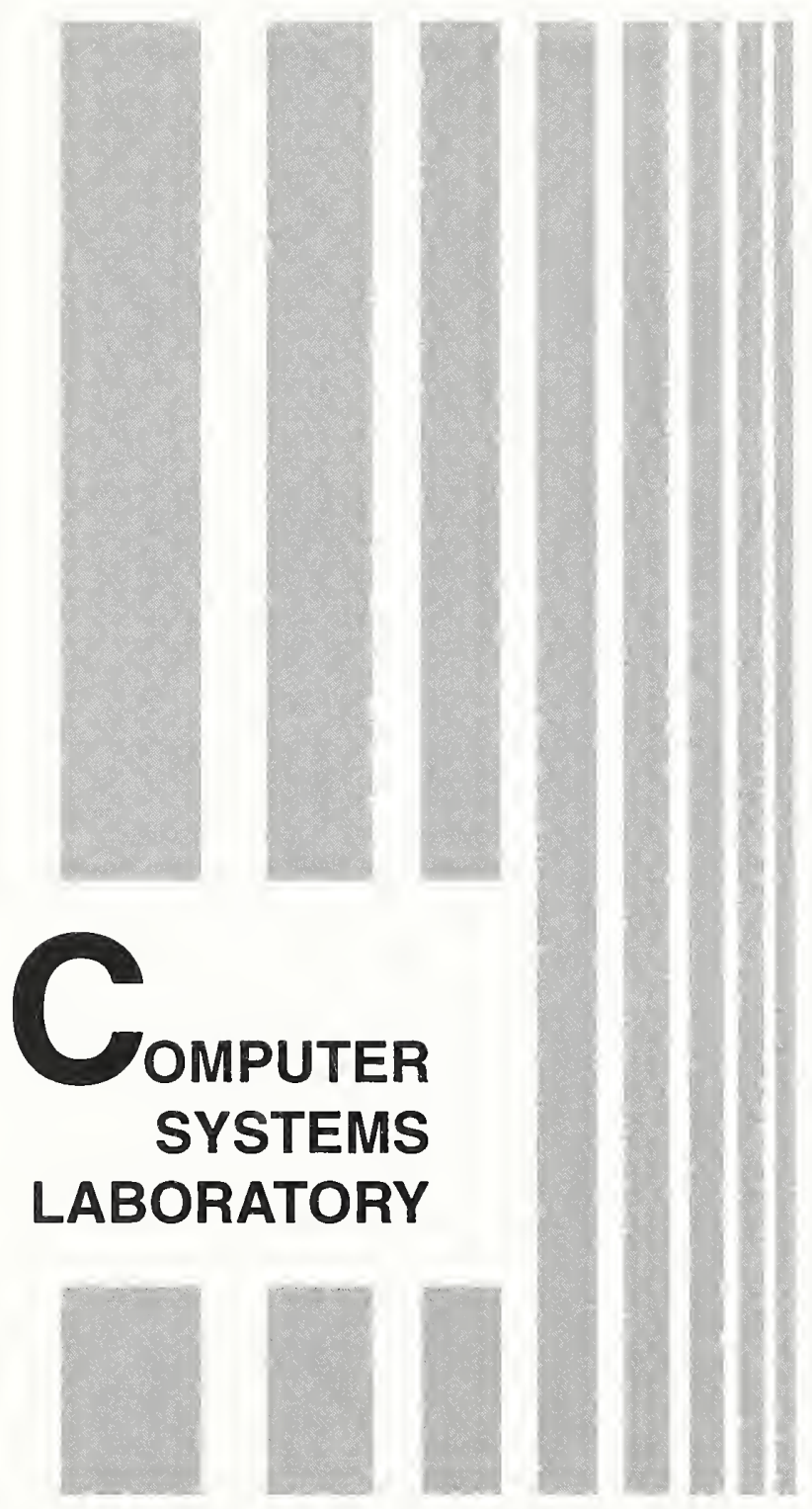





\section{DIRECTOR'S FOREWORD}

The pattern of change that has marked the development and use of information technology over the past few decades continues today. Changes in the tech-

nology itself, in the management of the technology, and in the supporting infrastructure for the technology are having profound influences on many organizations' activities. This report on the Computer Systems Laboratory (CSL) in 1991 discusses our work to assist private- and public-sector organizations in managing these changes for the benefit of their customers and programs.

CSL at the National Institute of Standards and Technology (NIST) is responsible for developing standards, guidelines, and test methods, and for providing research and technical assistance on computer and related telecommunications systems. In conjunction with users and industry, CSL is addressing the development of standards, information technology architectures, and conformance tests needed to improve information management in this decade.

Changes in the 1990s.

Identifying User Requirements.
In the 1990s, we can expect that changes in technology will result in many more activities being automated, decentralized, and distributed geographically throughout the enterprise. Increased processing power, faster data networks. high-capacity data storage, expert systems, and neural networks are some of the technologies that will be available.

To manage the technology, organizations are paying greater attention to the strategic value of information systems. Information technology architectures are being refined and changed to focus on support to the business of the enterprise. Some organizations are responding to change and their need to stay competitive by turning over their computing and telecommunications systems to service providers to run for them.

"Open computing systems" have become a requirement of many users. Users are looking to open systems to provide increased flexibility, more choices, and capabilities to do new functions. Open systems have been defined in many ways, but from the user's perspective, open systems allow organizations to develop integrated systems composed of computer and communications products that are acquired from a variety of sources, and to move applications software developed for different systems from one system to another. In the past. it has not been possible to do this as the users were locked into a specific vendor(s). Today no single vendor can supply the systems to meet the broad diversity of user requirements.

Standards have never been more important, but many of the needed standards for open systems are not available. Those standards that are available come from a variety of sources and are often not totally integrated or well defined. Some standards are being developed by national and international standards groups; some are being established by vendors working together: and others are being created through the marketplace. 
To get the needed standards, users are beginning to identify their common requirements for open systems. This past year, for example, the Standards for Open Systems group, comprised of information technology executives of American Airlines, DuPont, General Motors, Kodak, McDonnell Douglas, Merck, Motorola, 3M, Northrop, and Unilever agreed that they had a common need to accelerate the commercial availability of open systems based on vendor-neutral standards. The group developed an influential statement of strategic direction calling for products based on open systems specifications.

Requirements for open systems are arising in other parts of the world as well. Governments such as the UK and Canada, the Commission of the European Community, and an international group representing government procurement authorities are spearheading efforts to adopt open systems architectures. Worldwide acceptance and commitment to open systems will be essential in stimulating the development of cost-effective products and in providing worldwide markets for U.S. computer vendors.

Last year, we established a Federal Open System Users Council (FOSUC) as a forum for federal organizations concerned with open systems issues. FOSUC members identified their high-priority standards-related issues affecting agency missions and application areas. The issues included: electronic mail and electronic data interchange applications; implementation, migration, and expansion of the Government Open Systems Interconnection Profile (GOSIP): and data management and storage.

Open systems are becoming part of the strategies of many agencies, especially the Department of Defense which has implemented a Corporate Information Management program to improve the development, acquisition, and operations of information technology systems. A memorandum in May 1991 signed by Paul A. Strassmann, Director of Defense Information, said: "The Department is committed to establishing an open systems environment for its information systems. This open systems environment will have a technical architecture based exclusively on Federal standards for open systems."

Open systems reflect dramatic shifts in the computer industry and in user options, and will continue to be a focus of our programs over the next few years. We will be concentrating on the standards and specifications needed to give users flexibility in the development of systems, and in planning for the migration to open systems.

Another important area is the development of standards and technology to profor Open Systems. tect information from unauthorized modification, undetected loss, and unauthorized disclosure. Our computer security activities are covered in detail in this report, but I wanted to highlight a new collaborative effort with the National Security Agency (NSA) and industry users to develop a federal standard for specifying computer security requirements in open systems environments, including distributed applications such as Electronic Data Interchange (EDI). This work draws on the current Trusted Computer System Evaluation Criteria ("Orange Book") developed by NSA. 
Trusted systems are the computer and network systems that have security controls built into products, and have been used for some time to protect the confidentiality of data. The new criteria will extend the concept of trusted technology to include other protective mechanisms: assuring the integrity of data and processes; controlling access to network services, customer equipment, and data; and assuring the availability of data, systems, applications, and services for processing when needed.

High Performance Computing.

Response to Customer Needs.
Also important over the next few years will be research and development efforts for advanced computers, high-capacity and high-speed networks, and electronic data bases. This activity is supported by the High Performance Computing Act of 1991 and the High Performance Computing and Communications Program of the Office of Science and Technology Policy. Planned as an interagency activity with close cooperation between federal agencies, industry and academe, this activity is expected to accelerate the commercial availability of the next generation of high-performance computers and networks.

Total Quality Management programs establish a goal of customer satisfaction which can be applied to an organization's internal operations and the support that the organization's information systems give to programs and operations. Information managers can contribute to the quality process by understanding their customer's needs and developing improvement programs for their activities to advance the business of the organization.

Just as information managers need to listen to their customers, so does CSL need to listen to its customers. I believe that interactions between users, vendors, and the government on open systems, security, high-performance computing, and other issues will be beneficial to all of us, and I invite a continuing dialogue.

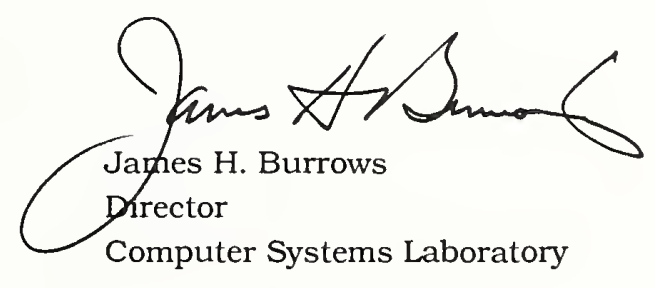





\section{CONTENTS}

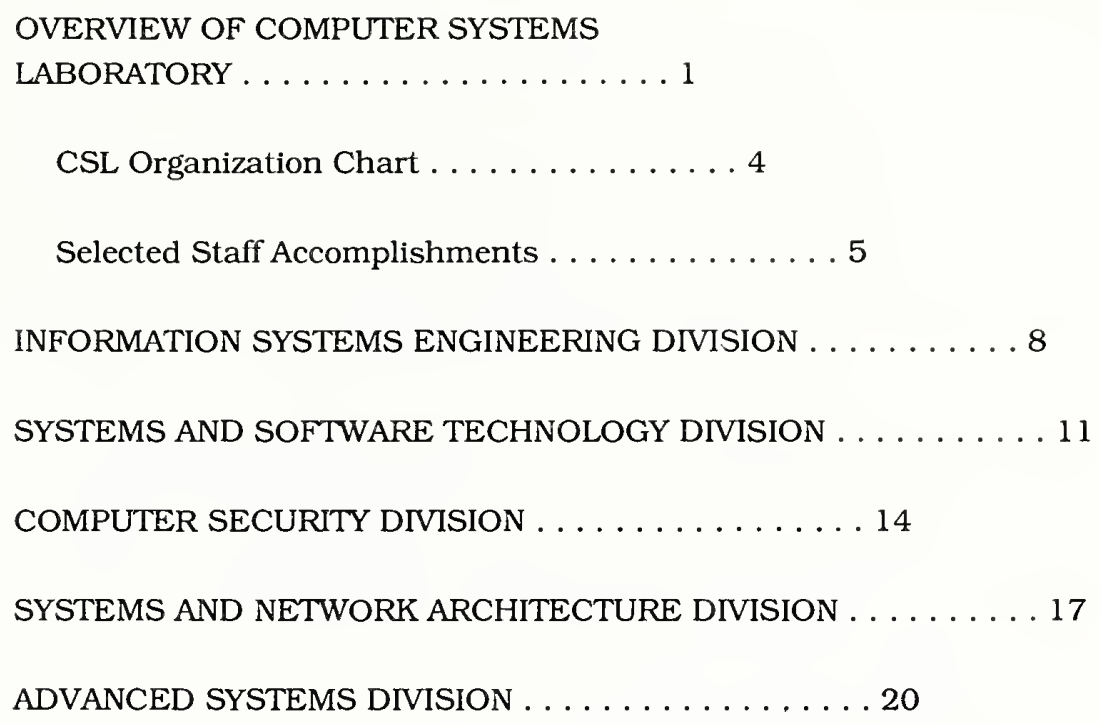





\section{OVERVIEW OF COMPUTER SYSTEMS LABORATORY}

As one of the major science and engineering research components of the National Institute of Standards and Technology (NIST), the Computer Systems Laboratory (CSL) plays a significant role in supporting government and industry by advancing the development and implementation of computer and telecommunications systems technology. CSL programs and research are mandated by the Brooks Act (Public Law 89-306), the Computer Security Act of 1987 (Public Law 100-235), and the Omnibus Trade and Competitiveness Act of 1988 (Public Law 100-418).

Program Activities and Goals.

Open Systems.

Focus on Research.
CSL develops standards, guidelines, and test methods for computer systems and networks, conducts research, and provides assistance and advisory services to the information systems communities of the federal government and industry. The goal of these activities is to provide application portability, interoperability, and security across different systems and networks, and to advance the development and use of high-performance computer and communications capabilities. CSL programs support key Department of Commerce goals of promoting international trade, speeding commercialization of new technologies, and providing technical leadership in the development of new standards and measurement methods.

CSL supports the development of technology and standards for open systems, a common vision of users in both public and private sectors. No single vendor is able to supply the systems to meet the diversity of user requirements. Enterprise-wide needs for common application architectures, communications, and networks are moving organizations away from proprietary systems and standalone applications of computers to greater integration of functions and increased exchanges with external organizations. Users need off-the-shelf hardware, software, and telecommunications products that will interoperate, and the ability to move data, applications, and people skills from one system or environment to another.

To support the development and implementation of complex open computer systems and networks, CSL continued its laboratory-based research programs focusing on computer security, software engineering, data management, data communications, and advanced systems. The development of test and measurement methods to evaluate conformance of products to standards and the interoperability of the many components in today's computer systems remain a high priority. Transferring technology to government and industry completes the research cycle. 
Interactions with Government and Indiustry.

Computer Security.

Organization and Resources.
Providing advice and assistance to the public and private sectors remained an important part of CSL's mission. Many organizations sought technical solutions to complex problems in a broad range of computer and telecommunications program areas. Some collaborations continued from previous years, while other cooperative ventures were initiated during 1991. Collaborative efforts benefit all organizations involved, and significantly advance the trend away from proprietary products and services to an open systems environment.

Open systems must also be secure systems. The Computer Security Act of 1987 strengthened and reaffirmed CSL's role in protecting vital data in federal computer systems and networks. Activities of our computer security program included proposed and revised Federal Information Processing Standards (FIPS), guidance on computer security topics, and visits to federal agencies with the Office of Management and Budget and the National Security Agency to gain management support for computer security. Other activities included training, publications, conferences, and sponsorship of the Federal Computer Security Program Managers Forum and the Computer System Security and Privacy Advisory Board established by the legislation.

CSL's work is carried out in five technical divisions: Information Systems Engineering Division, Systems and Software Technology Division, Computer Security Division, Systems and Network Architecture Division, and Advanced Systems Division. Our professional staff consisted of computer scientists, computer specialists, electrical and electronic engineers, and mathematicians. Staffing resources in FY 1991 included 231 full-time-equivalent employees of which $75 \%$ were professional and technical staff and $25 \%$ were administrative support personnel. In addition to CSL staff, about 26 research associates, guest scientists, and faculty appointments enhanced our research program. A total of 41 cooperative research projects with government, industry, and academia were in place in FY 1991.

Funding for CSL programs in FY 1991 consisted of $\$ 12.3$ million from the N1ST Congressional appropriation (STRS), including $\$ .7$ million in N1ST-supported competency funding and \$14.4 million in reimbursable funds, mostly for direct technical assistance from other federal agencies. About 37 organizations in government and industry received reimbursable technical support from CSL in FY 1991. The Department of Defense, the General Services Administration, and the National Aeronautics and Space Administration are representative of federal agencies that used our resources to solve technical problems. 
Sharing Information

Sharing information and technology with government, business, academia, and and Technology. the public is a primary goal of our organization. CSL publishes a variety of documents including FIPS, guidelines, computer systems and computer security reports on research and tests, a quarterly "CSL" newsletter, and a CSL bulletin series published about eight times a year on specialized topics of interest to the information systems community. See the Technology Transfer section for a list of FIPS and other publications currently available for sale through the Government Printing Office (GPO) or the National Technical Information Service (NTIS). A variety of conferences and workshops are sponsored and hosted by CSL throughout the year, and our staff members are invited to address many federal and private organizations.

CSL maintains four electronic bulletin boards to share information with computer users with dial-up capability. Bulletin boards offer information on computer security, data management, Open System Interconnection (OSI) activities, and Integrated Services Digital Network (ISDN). Instructions for accessing bulletin boards appear in Technology Transfer.

Technical highlights of our five divisions follow. 


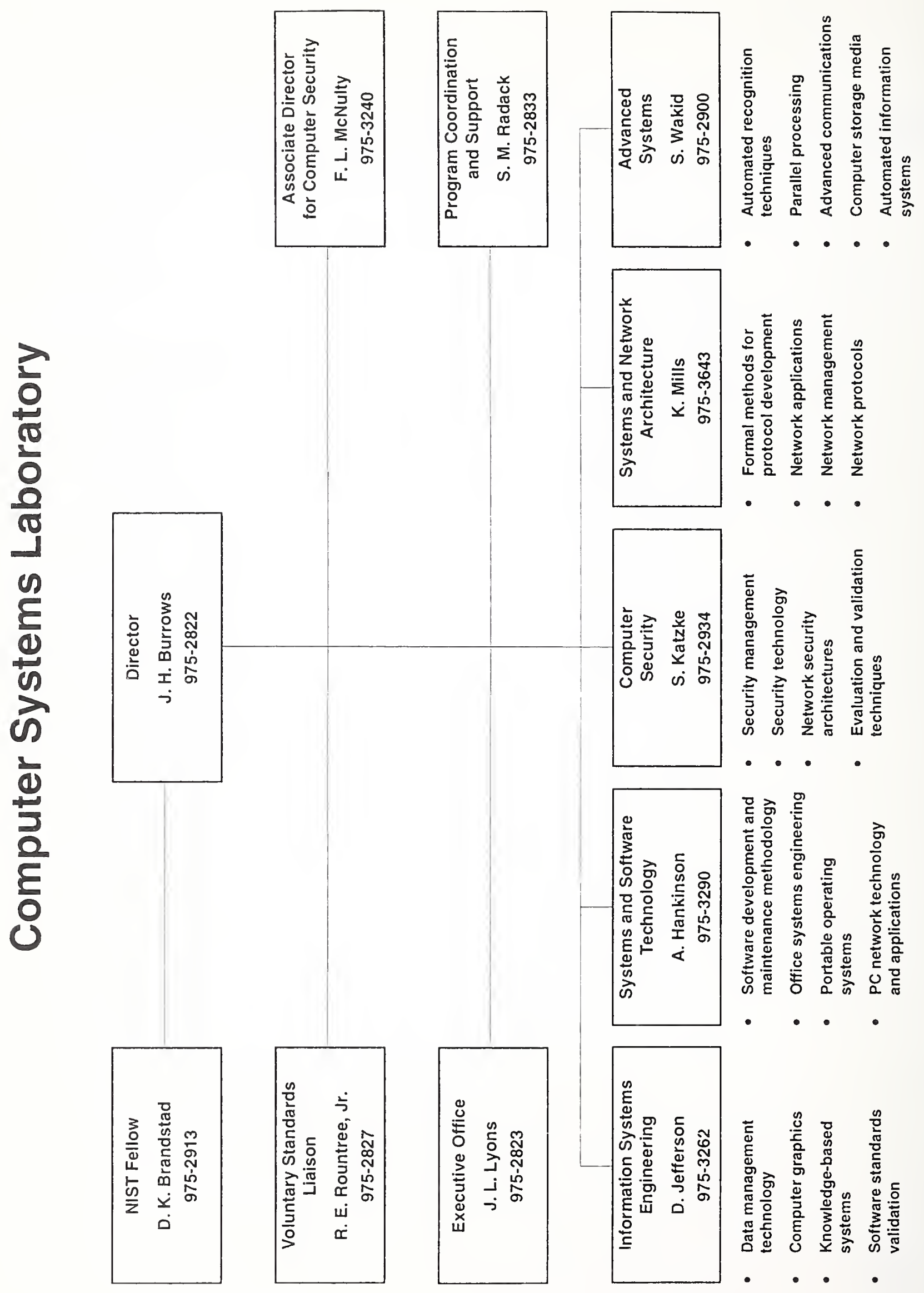




\section{SELECTED STAFF ACCOMPLISHMENTS FY 1989 - FY 1991}

Department of Commerce awards for major contributions to Department programs were presented to:

Allen L. Hankinson - Silver Medal (1991)

David K. Jefferson - Silver Medal (1991)

Roger J. Martin - Silver Medal (1989)

Miles E. Smid - Silver Medal (1989)

Barbara L. Blickenstaff - Bronze Medal (1990)

David E. Cypher - Bronze Medal (1991)

Gary E. Fisher - Bronze Medal (1991)

J. Elaine Frye - Bronze Medal (1989)

Irene E. Gilbert - Bronze Medal (1991)

David R. Kuhn - Bronze Medal (1990)

Candice E. Leatherman - Bronze Medal (1989)

Charles L. Sheppard - Bronze Medal (1990)

Joan M. Sullivan - Bronze Medal (1989)

David Hui-Yang Su - Bronze Medal (1989)

James H. Burrows was awarded the 1991 IRM Leadership Award by the Association for Federal Information Resources Management (AFFIRM).

James H. Burrows received the 1991 Federal Office Systems Exposition (FOSE) Award for leadership in standards development for computing, telecommunications, and computer security.

James H. Burrows received the Distinguished Presidential Rank Award for extended exceptional performance in government.

James H. Burrows was inducted into the Government Computer News Hall of Fame. 
James H. Burrows, Dennis K. Branstad, Kevin L. Mills, and Shukri A. Wakid received Federal 100 awards from Federal Computer Week for significant contributions to the government systems community in 1990.

James H. Burrows, Allen L. Hankinson, and Dennis D. Steinauer received the Federal 100 Reader's Choice Awards from Federal Computer Week for 1989.

F. Lynn McNulty, Patricia Edfors, Edward Roback, Vicky Howard, and Arlene Carlton, Office of the Associate Director for Computer Security, were recognized by Government Workplace for their governmentwide efforts in promoting effective computer security management.

Allen L. Hankinson was elected to the UniForum Board of Directors for a twoyear term beginning July $1,1991$.

Kevin L. Mills received the Interagency Committee on Information Resources Management 1991 Award for Management/Administrative Excellence for effective leadership in the federal systems community in advancing the acceptance of Open Systems Interconnection (OSI) standards.

Kevin L. Mills was elected as a senior member in the Institute of Electrical and Electronics Engineers (IEEE).

Kevin L. Mills was appointed to represent CSL on the new Federal Networking Council.

Robert Rountree Jr. has been elected, for a three-year term beginning in 1991, Chair of JTC1 TAG, the American National Standards Institute (ANSI) technical advisory group for the international standards committee ISO/IEC Joint Technical Committee 1 (Information Technology).

Michael D. Hogan has been elected Vice Chair of the Information Systems Standards Board (ISSB) for a two-year term beginning in 1992.

Donna Harmon and Gerald Candela received the 1990 R\&D 100 Award from Research \& Development magazine for a fast information retrieval system.

Donna Harmon and Gerald Candela were the recipients of the 1991 Journal of the American Society for Information Science (JASIS) Best Paper Award for "Retrieving Records from a Gigabyte of Text on a Minicomputer Using Statistical Ranking."

Robert J. Carpenter, Alan Mink, George Nacht, and John Roberts received the Allen V. Astin Measurement Science Award for their contributions to the science of measuring the performance of multiprocessor computer systems. 
Roger J. Martin received the Interagency Committee on Information Resources Management Award for Technical Excellence in 1989 for outstanding contributions to the federal information resources management community.

Miles E. Smid received the Award for Technical Excellence from the Interagency Committee on Information Resources Management in 1990 for his contributions to the federal information resources management community, particularly in computer security.

Leonard J. Gallagher received the Interagency Committee on Information Resources Management Award for Technical Excellence in 1989 for outstanding contributions to the federal information resource management community.

Miles E. Smid received the Commissioner's Citation from the Department of the Treasury in 1989 for outstanding contributions to Treasury payments systems.

Mark Skall was reappointed to the National Computer Graphics Association Board of Directors.

Gordon Lyon was appointed Chairman of the NIST Research Advisory Committee for 1991 .

Leonard J. Gallagher was selected by the Oracle Corporation in 1990 as the grand prize winner of its "Unleash the Genius" contest for his implementation of a hypertext query facility.

Edward Roback received an Unsung Hero in Computer Security Award by FedSecurity ' 91 and Federal Computer Week.

Henry Tom was elected as the government representative on the Board of Directors of the National Computer Graphics Association.

Fernando L. Podio is chairman of the NIST/NASA Working Group for the Development of Test Methods and Specifications for $356 \mathrm{~mm}$ Ruggedized Rewritable Media.

Fernando L. Podio is chairman of the Working Group on Monitoring and Reporting Techniques for Error Rate and Error Distribution in Optical Disk Systems. 


\section{INFORMATION SYSTEMS ENGINEERING DIVISION}

The Information Systems Engineering Division develops standards and provides technical assistance to government and industry in data administration, data management technology, computer graphics, and software standards validation.

Data Administration.

CSL continued to support the voluntary standards efforts concerning the Information Resource Dictionary System (IRDS), both at the American National Standards Institute (ANSI) level and at the International Organization for Standardization (ISO) level. The current ANSI IRDS standard was adopted as Federal Information Processing Standard (FIPS) 156 in 1989, and CSL continues to play an active part in the development of several new optional modules for this standard. Further, the development of a conformance test to cover FIPS 156 is in process. CSL also serves as the technical editor for the document that is being developed by the voluntary standards community that will provide the requirements for the development of the next generation of IRDS, now referred to as IRDS2. These future efforts on IRDS2 will focus on utilizing the IRDS as a mechanism to integrate the results produced through the use of ComputerAided Software Engineering (CASE) tools throughout an application system's life cycle.

The Data Administration Management Association (DAMA) annual symposium was again cosponsored by CSL. This year's symposium focused on managing an organization's data resources from the first realization of the need for the data through the entire life cycle of that data.

CSL established new working agreements this year with the Department of Veterans Affairs, the Department of Education, and the Environmental Protection Agency. In addition, previous agreements with the Department of Defense (DoD) Corporate Information Management (CIM) and the Internal Revenue Service were continued. These agreements cover topic areas such as tool integration and the establishment of data administration policy for an organization that takes advantage of standards to achieve an open systems environment for data administration.

Data Management Technology.
In an ongoing effort to help users and vendors determine compliance with FIPS 127-1, Database Language SQL, CSL released Version 2.1 of the NIST SQL Test Suite in June 1991. Version 2.1 adds the Embedded Ada and Module Language Ada test suite types to the existing test suite types for C, COBOL, Fortran, Pascal, and Interactive SQL. Sixteen SQL Test Suite licenses were added this fiscal year. Since the NIST SQL Test Suite was released, over 95 SQL Test Suite licenses have been purchased for all versions of the test suite. The NIST SQL Test Suite is being used in the NIST SQL Testing Service since SQL testing began in April 1990. A Registered Validation Report documents each validation. 
Research efforts in expert systems technology and distributed database technology produced two guides: NIST Special Publication 500-185, Guide to Design, Implementation, and Management of Distributed Databases, and NIST Special Publication 500-188, Guide to Expert Systems Building Tools for Microcomputers.

A research project in hypertext completed its third year in collaboration with the Systems and Software Technology Division. Major accomplishments in this project include CSL-sponsored hypertext workshops as well as the knowledge and experience gained in the integration of hypertext technology with expert systems, database, graphics, and publishing. Project members gave presentations at external workshops and conferences and produced articles, reports, and reviews. This laboratory-based research is carried out in the Multimedia Systems and Database Laboratories.

Under an interagency agreement, CSL continued its assistance to the DoD Computer-aided Acquisition and Logistic Support (CALS) project in the application of SQL and other data management standards to CALS requirements. NISTIR 4494, SQL3 Support for CALS Applications, resulted from these efforts. CSL also provided consulting services to the Department of the Army for the Sustaining Base Information Services (SBIS) and to the Defense Advanced Research Projects Agency (DARPA) for object database technology.

Computer Graphics. Conformance testing in computer graphics remained a high priority. CSL fosters the protection of federal investment in computer graphics by developing conformance tests which determine whether implementations conform to FIPS and by establishing test services to administer conformance tests. Working closely with European colleagues ensures harmonization in computer graphics testing.

FIPS 120, Graphical Kernel System (GKS), added a requirement for implementations to be tested in order to be considered for procurement by federal agencies. The CSL test service for GKS implementations conducted its first onsite validation and issued its first certificate of conformance. CSL sold nine copies of Version 1 of the Programmer's Hierarchical Interactive Graphics System (PHIGS) Validation Test Suite. Version 2 will be completed in spring 1992 at which time a PHIGS Testing Service will commence. CSL also established a Computer Graphics Metafile (CGM) Testing Service to determine conformance to FIPS 128, CGM, and the CALS Application Profile (MIL-D-28003).

On the international scene, CSL monitored the work taking place in Europe on Computer Graphics Interface (CGI) conformance testing. Also of note is CSL's designation by the International Organization for Standardization/International Electrotechnical Commission (ISO/IEC) as the Registration Authority for ISO Registration of Graphical Items. In this capacity, CSL developed the ISO Register which currently has 16 Linetypes, 19 Hatchstyles, 5 Escapes, 5 Marker Types, and 4 Generalized Drawing Primitives. To date four copies of the ISO Register have been distributed.

Geographic Information Systems (GIS) continued as a major growth activity. 
Software Standards Validation.
Testing programming language compilers for conformance to FIPS programming language standards and Federal Information Resources Management Regulations (FIRMR) continued to be an important service. In addition to providing validation services for programming languages COBOL (FIPS 21-3), Fortran (FIPS 69-1), Pascal (FIPS 109), and Ada (FIPS 119), CSL established a new validation service for the programming language MUMPS (FIPS 125). The MUMPS validation service was developed through an interagency agreement with the Department of Veteran Affairs which funded the MUMPS validation research.

In FY 1991, CSL selected a test suite for testing conformance to FIPS 160, C. Approved by the Secretary of Commerce in March 1991, FIPS 160 became effective September 30, 1991. A validation service for FIPS 160 is planned for 1992.

CSL received a large increase in requests for validation services for FIPS $127-$ 1, Database Language SQL. In FY 1991, 25 database language processors from 6 vendors were validated for conformance to FIPS 127-1 compared to one validation the previous year.

During FY 1991, programming and database validation services were provided to 45 private-sector companies and one government agency for a total of 171 validations. The total number of programming and database language processors currently validated as of September 30, 1991, is 347 .

CSL continued to publish quarterly the Validated Products List which is a collection of registers listing implementations that have been validated for conformance to FIPS. In addition to listing validated products of COBOL, Fortran, Pascal, Ada and SQL, the publication expanded to include lists of validated products for GKS, Portable Operating System Interface for Computer Environments (POSIX), and Government Open Systems Interconnection Profile (GOSIP). This publication is now sold through the National Technical Information Service on a subscription basis.

To facilitate the worldwide conformance testing effort, CSL sponsored a 5th International Workshop on Harmonizing Conformance Testing of Computer Language Standards. Experts from the United Kingdom, France, Italy, Germany, Japan and the U.S. participated in the workshop. The attendees researched common areas of agreement among testing laboratories and certification authorities for harmonizing validation testing activities. 


\section{SYSTEMS AND SOFTWARE TECHNOLOGY DIVISION}

The Systems and Software Technology Division develops standards and provides assistance in software engineering and office systems engineering to federal agencies and industry organizations. Technical activities during 1991 included the following:

Application Portability Profile (APP) Guide.
CSL published a key document in April 1991. NIST Special Publication 500187. Application Portability Profile (APP) The U. S. Government's Open System Environment Profile OSE/ 1 Version 1.0, defines an open system environment (OSE) framework by describing the information technology (IT) services, protocols, interfaces, and data formats needed by the U.S. government to support a broad range of federal applications. For each of the seven service areas included in the APP, standards and other specifications are recommended for use in developing and acquiring systems within government agencies. The APP Guide, as it has become known, also impacted many private-sector organizations who adopted the recommended specifications in their internal environments to provide better management and control of IT resources.

As open systems evolve, the APP will encompass new technology and capabilities upon which consensus can be built. The goal of open systems and the APP is the recommendation of a complete set of specifications for all OSE services in the form of international standards. The current state of standards will not allow IT users to realize the goal of open systems for some time. In the interim, the APP Guide provides recommendations on OSE specifications and evaluates these specifications based on criteria such as maturity, stability. completeness, level of consensus, and other factors that allow individual agencies to make informed choices in the selection of products and services.

With the APP Guide, the U.S. government now has a framework for organizing and describing standards and information technology specifications, as well as a base document for the discussion of open systems. The Application Portability Profile/Open System Environment (APP/OSE) Users' Forum met twice this year to provide a sounding board for users, vendors, and implementors on APP/OSE issues. These forums are instrumental in distributing information concerning OSE developments in the federal government and in providing guidance to federal agencies. In a related area, the new NIST Special Publication 500-184, Functional Benchmarks for Fourth Generation Languages, assists managers selecting a fourth generation language (4GL) to determine how well a particular 4GL will meet organizational, application, and user requirements. 
POSIX Conformance Testing.

High Integrity Software.
A testing program was started for FIPS 151-1, POSIX. POSIX promotes the portability of application software at the source code level, between computer systems from multiple vendors. Seven accredited POSIX testing laboratories were announced and the first group of NIST POSIX validated products was developed in May. Each validated product was tested by a laboratory accredited by the National Voluntary Laboratory Accreditation Program (NVLAP), using the NIST POSIX Conformance Test Suite (NIST-PCTS:151-1) for FIPS 1511. The test results were validated by CSL and a Certificate of Validation issued. The number of POSIX validated products continues to grow and is published quarterly in CSL's Validated Products List. The October 1991 CSL Bulletin describes the NIST POSIX testing program in detail.

Most federal procurements will require submission of a certificate of validation or proof that a vendor product conforms to FIPS 151-1. Validated products give a degree of assurance to the government that the desired portability and interoperability will be attainable.

High integrity systems are those systems whose failure can result in loss of property, personal injury, accidental death, environmental harm, and diminished confidence in the business and social infrastructure. CSL initiated a Lecture Series on High Integrity Systems and sponsored five lectures on topics such as software engineering from a systems perspective, cleanroom engineering, and software process assessment. The lecture series targets federal and industry managers, technical staff, and users.

A Workshop on High Integrity Software was held at NIST on January 22-23, 1991, to explore the development of a framework for standards to assure that critical software can be trusted to work as required. The workshop proposed a template for describing methods and techniques for assuring high integrity software and proposed topics for further study, including cleanroom engineering, traces, and some formal specification languages. NIST Special Publication 500190, Proceedings of the Workshop on High Integrity Software, Gaithersburg, MD; Jan.22-23, 1991, documents the results of the workshop. Results of a Forum on Standards for High Integrity Software held at NIST in June 1991 are contained in NISTIR 4656.

CSL again cosponsored the Sixth Annual Conference on Computer Assurance, COMPASS ' 91 , an annual event focusing on the assurance of the integrity of computer systems and information resources. 
Integrated Software Engineering Environments.
The need for high-quality software is closely associated with the need to improve productivity in the development and evolution of that software in integrated software engineering environments (ISEE). An ongoing series of invitational ISEE workshops continued to develop a reference model and to propose interfaces for fully integrated software engineering environments which support software products and processes throughout the software life cycle.

The workshop works closely with other efforts in software engineering environments including the European Computer Manufacturers' Association (ECMA), the Navy's Next Generation Computer Resources (NGCR), the Defense Advanced Research Projects Agency (DARPA), the Ada Joint Program Office, the International Workshop on Computer-Aided Software Engineering, and various Department of Defense, National Aeronautics and Space Administration, Institute of Electrical and Electronics Engineers, and industry efforts. CSL and ECMA agreed to publish a joint "Reference Model for Frameworks of Software Engineering Environments" Technical Report.

In September, CSL proposed the use of the ECMA PCTE (Portable Common Tool Environment) specification as the base document in the development of tool interface specifications for software engineering environments. This proposal, coupled with an aggressive effort to bring together the many different groups working on aspects of tool interfaces for an ISEE, led to an increased coalescence of efforts around the CSL ISEE workshops.

Multimedia Systems. Office systems engineering activities focused on developing and implementing computer-based tools to enhance productivity. Since many tools do not work well together, CSL efforts centered on the use of open system concepts to integrate computer-based office tools.

Researchers in the Multimedia Systems Laboratory investigated how document standards can coexist and how users can benefit from the best of each standard. NISTIR 4560, Government Document Processing Requirements Report, describes significant activities impacting on the harmonization of standards within the electronic publishing area. It focuses on a list of user requirements resulting from a workshop on Electronic Information Exchange Standards and other CSL efforts to bring about the harmonization of electronic publishing standards.

In support of the Department of Defense Computer-aided Acquisition and Logistic Support (CALS) program, CSL encoded the Office Document Architecture (ODA) Document Application Profile (DAP) in the Standard Generalized Markup Language (SGML) to illustrate similarities between the two standards and to provide a common SGML/Abstract Syntax Notation One (ASN.1) profile. NISTIR 4547 describes the two international standards and discusses the methodology involved in performing an SGML encoding.

In collaboration with the Information Systems Engineering Division, researchers continued a joint project on hypertext and hypermedia technologies. As part of that effort, a new Hypermedia Lecture Series was initiated. Hypermedia technologies permit the integration of searching, linking, and multimedia presentations using optical storage and networked systems. 


\section{COMPUTER SECURITY DIVISION}

The Computer Security Division develops standards and guidance for the costeffective security of information resources in computer and telecommunications systems. In response to the mandate of the Computer Security Act of 1987 , CSL continued to provide guidance, assistance, research, and technical support to federal agencies and industry in computer security.

\section{Proposed FIPS for Digital Signature Standard (DSS).}

Security Requirements for Cryptographic Equipment.

Risk Management.
In August 1991, CSL announced a proposed Federal Information Processing Standard (FIPS) for DSS for use by federal agencies. The proposed standard specifies a public-key-based digital signature algorithm (DSA) appropriate for federal digital signature applications. The DSS uses a public key to verify to a recipient the integrity of data and the identity of the sender of the data. The DSS can also be used by a third party to ascertain the authenticity of a signature and the data associated with it.

The proposed standard adopts a public-key signature system that uses a pair of transformations to generate and verify a digital value called a signature. Requirements for public-key cryptography have expanded as organizations have developed electronic mail systems and electronic funds transfer applications. In such settings, an electronic equivalent of the handwritten signature may be desirable.

To help organizations use the latest security technology for the protection of information, CSL initiated a revision of FIPS 140, a standard that establishes the physical and logical security requirements for the design and manufacture of Data Encryption Standard (DES) equipment. The revision will be reissued as FIPS 140-1, General Security Requirements for Cryptographic Modules.

Defining four security levels for cryptographic modules, FIPS 140-1 will incorporate cryptographic algorithms and functions specified in related FIPS. The revised FIPS will be an umbrella standard which will provide a framework under which cryptographic standards will be implemented in products. The revision includes the development of requirements for software assurance and verification to be applied to cryptographic equipment.

Also developed was a test assertions document to be used as the basis for CSL's planned validation program for FIPS 140-1 implementations. Commercial laboratories will be recognized under NISTs National Voluntary Laboratory Accreditation Program (NVLAP) to do conformance validation for FIPS 140-1. Testing for the higher security levels will require the use of formal methods of software specification. CSL encourages the use of formal methods to enhance the development of quality software products.

Risk management requires computer system managers to identify risks to their systems and to develop cost-effective means of reducing risks. Risk management implies good security planning based on full awareness of the issues, the constraints, and the resources available. 
To assist those responsible for federal risk management, CSL and the National Security Agency's National Computer Security Center (NCSC) cosponsor the Risk Management Laboratory at NIST's Gaithersburg site. The laboratory serves as an information resource for federal agencies considering the use of automated risk management software packages and advances understanding of risk management. More than 30 risk management packages have been installed in the laboratory and demonstrated to interested federal agencies. CSL and NCSC cosponsored the annual international workshop for risk model builders to develop a consensus on a conceptual framework for risk analysis.

Trusted Systems Technology.

POSIX Security.
CSL and NSA initiated a cooperative effort to develop a comprehensive set of FIPS to specify requirements for the design, development, and assessment of trusted information products and systems. These are computer and network systems that have high-quality and reliable security controls built into products. The cooperative effort will develop new criteria for trusted systems to replace the current Trusted Computer System Evaluation Criteria (TCSEC) that were developed by NSA to evaluate the ability of systems to protect the confidentiality of data. The new criteria will add capabilities for evaluating other security controls, such as assuring the correctness of data and processes, controlling access to services and data, and assuring the availability of data, systems, applications, and services.

A related project is concerned with the development of standardized and more readily available processes for evaluating and certifying security products for compliance with the new criteria. The evaluation process is key to increasing the confidence of the user community to trust such products to do what they are required to do. Vendors need a flexible and well-defined process that will help them develop security products to meet all levels of assurance.

To support an international approach to evaluating trusted systems, CSL is working with the Commission of the European Communities (CEC) which has developed its own Information Technology Security Evaluation Criteria (ITSEC) The goal of this collaboration is to develop a common basis for product evaluation and to avoid multiple testing requirements that would be expensive for both users and vendors. CSL and the CEC are in the process of formalizing their information security collaboration by completing a joint statement of strategic direction.

CSL chaired the voluntary industry working group which is defining the security requirements for basic internal controls needed for POSIX-like operating systems. POSIX is the Portable Operating System Interface for Computer Environments (FIPS 151-1, adopting IEEE 1003.1-1988). The P1003.6 standard. POSIX Security Extensions, provides a set of application program interfaces (APIs) to basic security functions, including access control lists, audit trail mechanisms, privileges, and mandatory access controls. These standard security interfaces will enable the development of portable and interoperable trusted application programs.

Guest researchers from Bellcore work with CSL in both the POSIX security effort and in the development of new criteria for trusted systems. 
Security Labels for Open Systems.

Cooperative
Interactions.

\section{Sharing} Information.
CSL hosted its Second Invitational Workshop on Security Labels for Open Systems on April 9-10, 1991. The workshop focused on an initial draft for a planned FIPS on a Standard Security Label Format for the Government Open Systems Interconnection Profile (GOSIP) and Procedures for Registering Computer Security Objects. Security labels indicate sensitivity and the possible damage which may occur due to accidental or intentional disclosure, modification, or destruction of data. The Registration Procedures are related to the proposed FIPS and will apply to the development of a Computer Security Object (CSO) Register which will include items such as specifications of security domains, security labels, security algorithms, security techniques, and security support systems. The proposed FIPS is expected to be approved in 1992 .

Established by the Computer Security Act of 1987 as a government/industry cooperative endeavor, the Computer System Security and Privacy Advisory Board met four times in 1991 to investigate and discuss key computer security issues. CSL hosted five meetings of the Federal Computer Security Program Managers Forum this year to share experiences and information on mutual problems and possible solutions.

In another cooperative venture, CSL helped to organize and operate the Forum of lncident Response and Security Teams (FIRST). This collaboration of several government and private-sector organizations is designed to foster cooperation and information sharing regarding security incidents in constituent systems and networks. Also continued was the research activity that helps federal agencies better protect their computer systems from computer viruses and related threats.

In October 1991, CSL and NSA's National Computer Security Center cosponsored the 14th National Computer Security Conference in Washington, DC. The conference attracted more than 1,700 participants from government, academia, and industry who came from the U.S., Canada, Europe, Asia, and Australia. This year's theme, "Information System's Security: Requirements and Practices," highlighted the trend toward developing broadly based solutions for protecting information assets.

As part of a continuing effort to assist federal agencies, CSL republished the work of other federal agencies and industry organizations to provide for broad public dissemination of federally sponsored work, including: the National Aeronautics and Space Administration (NASA) Automated Information Security Handbook; the Department of Health and Human Services (HHS) Information Systems Security Handbook; and a glossary of computer security terminology. Also published were CSL Bulletins on the computer security roles of NIST and NSA; FlPS 140 - A Standard in Transition; Security lssues in the Use of Electronic Data lnterchange; and advanced authentication technology. Other publications of note include NIST Special Publications 800-2, Public-Key Cryptography. 800-3, Establishing a Computer Security Incident Response Capability (CSIRC), and 500-189, Security in ISDN. CSL's expanded Computer Security Bulletin Board System (BBS) continued to reach users with dial-up capabilities with a wealth of information on information systems security. 


\section{SYSTEMS AND NETWORK ARCHITECTURE DIVISION}

Programs in the Systems and Network Architecture Division address the development and standardization of Open Systems Interconnection (OSI), the development and application of automated protocol methods, and the advancement of technology for integrated, interoperable network management. OSI networks permit equipment and systems from different manufacturers to interoperate.

GOSIP. In April 1991, the Secretary of Commerce approved Federal Information Processing Standard (FIPS) 146-1, Government Open Systems Interconnection Profile (GOSIP), Version 2.0. FIPS 146, Version 1.0, was approved in 1988 and provided OSI protocols for Message Handling System (MHS) and file transfer. access and management (FTAM) functions. MHS allows a user to send or receive electronic mail; MHS evaluation guidelines are contained in NIST Special Publication 500-182. The July 1991 CSL Bulletin describes FTAM in detail, and NIST Special Publication 500-196. Guidelines for the Evaluation of File Transfer, Access and Management Implementations, assists users in determining which FTAM implementation best meets their requirements.

Version 2.0 of GOSIP provides protocols for additional functions and capabilities including Virtual Terminal Service, Office Document Architecture, Integrated Services Digital Network, End System-Intermediate System protocol, and user options for Connectionless Transport Service and Connection Oriented Network Service. NIST Special Publication 500-192, Government Open Systems Interconnection Profile Users' Guide, Version 2, assists federal agencies in planning for and procuring OSI. Future versions of GOSIP will incorporate additional applications. GOSIP is based on agreements reached by the OSI Implementors Workshop (OIW).

The OIW met four times in 1991. The workshop serves as an open forum where more than 200 computer manufacturers, vendors, and users worldwide meet to share ideas and experiences and to advance the technology and standardization of open systems. NIST Special Publication 500-183, Stable Implementation Agreements for Open System Interconnection Protocols, Version 4, Edition 1. December 1990, records stable implementation agreements of OSI protocols developed by organizations that participate in the OIW.

In June 1991, the OIW established a Technical Liaison Committee ad hoc Task Group on Open System Environment Expansion to explore issues and develop a proposal to expand the scope of the OIW to encompass the total Open System Environment (OSE). The Task Group, and later the OIW, endorsed a set of recommendations to enlarge the scope of the OIW while retaining its current focus of achieving interoperability among multiple vendors' systems. In addition to implementing the specific recommendations of the Task Group, the expansion of OIW activities into an Open System Environment will entail addressing the full range of interface issues found in a distributed computing environment. 
Conformance and Interoperability Testing.

Global Recognition of OSI.
During 1991, CSL initiated a collaboration with several major industry proponents for OSI. General Motors, representing the Manufacturing Automation Protocol (MAP) program, Boeing, representing the Technical and Office Protocol (TOP) program, the Electric Power Research Institute (EPRI) and Pacific Gas and Electric (PG\&E) representing the electric power industry's Unified Communications Architecture (UCA), and CSL, representing the GOSIP program, agreed to produce a common government and industry OSI specification. The resuIting specification, built on GOSIP Version 2.0, will become GOSIP Version 3.0, as well as the next versions of MAP, TOP, and UCA. The common specification will be called the Industry Government Open Systems Specification (IGOSS).

CSL established the GOSIP Testing Program to permit federal agencies to substantiate claims of GOSIP compliance. The on-line U.S. GOSIP Register Database (GRD) developed and maintained by CSL contains a set of registers including those for test suites, test systems (means of testing), conformance testing laboratories, tested products, and interoperability testing services. The May 1991 CSL Bulletin describes the GOSIP Testing Program in detail and gives instructions for accessing the GRD.

As part of a research program to advance OSI routing technology, CSL performed interoperability tests on equipment using the OSI Intermediate System to Intermediate System Intra-domain Routing Exchange Protocol. Five industry organizations participated in the cooperative testing program. Researchers documented the test scenarios and results to provide comments to implementors; to guide standards communities in refining base standards. implementors' agreements, and user group profiles; and to solicit reactions on developing testing and evaluation methods.

CSL continued to participate in OSINET, a cooperative government/industry research network with about 26 participants. The database containing the OSINET Testing \& Registration Service developed by CSL was transferred to the OSINET secretariat, the Corporation for Open Systems. NISTIR 4607, Test and Registration User's Guide, and NISTIR 4668. The OSINET Testing and Registration Service Functional Specification describe the OSINET service and database.

On May 6-8, 1991, CSL hosted a Worldwide Recognition of OSI Test Results Workshop which was cosponsored by the International Organization for Standardization (ISO) and the International Electrotechnical Commission (IEC). Attracting about 150 participants from Europe, Australia, the Orient, North and South Americas, the workshop addressed the technical procedures and criteria necessary for the achievement of equivalence of test results from OSI testing laboratories worldwide. The issues of harmonizing testing laboratory accreditation and OSI product certification received special emphasis. 
Electronic Data Interchange (EDI).

\section{Network Protocols.}

\section{Interoperable Network Management.}

To assist federal agencies in the use of EDI, CSL initiated an EDI program to promote the integration of this technology in an open systems environment. Sponsored by the Department of Defense, the Internal Revenue Service, and the General Services Administration, the program will produce generic EDI software which will be placed in the public domain. A generic set of EDI tools referred to as the EDI Transaction Set Development System (TSDS) will provide users with a tool for prototyping, testing, and using their transaction sets. CSL may initially apply these tools to proposed new transaction sets for securing EDI transactions. A prototype of EDI transmission software, primarily focusing on an EDI X.435 User Agent, will encourage users to experiment with the transmission of EDI transactions through X.400 networks.

The long-term goals of the EDI program are to provide tools for effectively testing commercial EDI products, to assist in the standardization of Application Program Interfaces (APIs) for EDI, and to develop an evaluation guidelines document that will assist users in selecting which EDI implementation, among several candidates, best meets user requirements. A FIPS for EDI was approved in 1991. The June 1991 CSL Bulletin discusses security issues in the use of EDI.

With support from the General Services Administration. CSL is deploying an X.500 pilot for the federal government. The pilot will transfer knowledge concerning a key technology, the OSI Directory, into government agencies. With the burgeoning use of data communications, it becomes more critical that the Directory be deployed to support naming, locating, and addressing resources.

Data from participating federal agencies was test-loaded into the CSL prototype X.500 implementation. Following deployment at NIST, the pilot was released to agencies which ran Directory System Agents linked to those at NIST. In keeping with the long-term objective of encouraging commercial implementation of X.500, CSL is considering how best to involve vendors in the pilot activity. A proposal for vendor participation is under development and is expected to be distributed in mid-1992.

In a related area, CSL concluded its collaborative effort with the Federal IRM Policy Council (FIRMPoC), an interagency group sponsored by the Office of Management and Budget, to provide members with electronic mail (E-mail) connectivity based on the CCITT X.400 series of recommendations. An interagency working group chaired by CSL developed the procedures for interconnecting FIRMPoC members through a standards-based E-mail facility on the FTS-2000 network. The project is now operational.

In July 1991, CSL proposed a FIPS adopting the Version 1.0 Government Network Management Profile (GNMP) for federal agency use. The GNMP specifies the common management information exchange protocol and services, specific management functions and services, and the syntax and semantics of the management information required to support monitoring and control of the network and system components and their resources. The GNMP builds on FIPS 146-1, GOSIP, and includes the GOSIP Version 2.0 by reference. The GNMP and GOSIP are interrelated and will cross-reference each other as required. CSL also continued to work with industry consortia to bring the GNMP into alignment with industry network management efforts. 


\section{ADVANCED SYSTEMS DIVISION}

Activities of the Advanced Systems Division focused on research and technical assistance to federal agencies and industry in parallel processing, data storage, distributed systems, automated recognition, and Integrated Services Digital Network (ISDN).

Parallel Processing. Researchers continued to seek an integrated approach to the measurement and characterization of computer performance. One approach focused on the performance evaluation of hypercube-like designs. Partially sponsored by the Defense Advanced Research Projects Agency, researchers studied the speed of communication which is important in the performance of programs for loosely coupled machines. NISTIR 4630, Performance Evaluation of Hypercube Applications: Using a Global Clock and Time Dilation, describes the separation of the communication component of a program, via a global clock, into two states: logical and physical delays. True measurements calibrate the indirect dilation method for a sharper, quantitative interpretation than otherwise possible. A "MULTIKRON" Very Large Scale Integration (VLSI) processor has been designed and tested by CSL and can now be used for the instrumentation of various parallel architectures.

Data Storage. Research in data storage technologies resulted in the production of a Standard Reference Material (SRM) for magnetic tape cartridges. NIST Special Publication 260-1 15, Standard Reference Materials: Calibration of NIST Standard Reference Material 3201 for 0.5 Inch $(12.65 \mathrm{~mm})$ Serial Serpentine Magnetic Tape Cartridge, describes the test system design and operation for the calibration of the NIST secondary standard reference tapes SRM 3201 for 0.5 inch $(12.65 \mathrm{~mm})$ 22 and 48 track serial serpentine magnetic tape cartridges. The SRM promotes the ability to interchange data both within and among various computer systems. When the media is designed and manufactured on the basis of a comparison to a known and accepted standard reference media, reliable interchange is assured.

A second research area focused on monitoring and reporting techniques for error rates and error distributions in optical disk systems. CSL hosted a workshop to identify user requirements and to propose an approach for documenting a Small Computer System Interface (SCSI) common command set for error rate monitoring and reporting. Workshop proceedings are contained in NIST Special Publication 500-198, Monitoring and Reporting Techniques for Error Rate and Error Distribution in Optical Disk Systems. Another new publication, NIST Special Publication 500-191. Test Methods for Optical Disk Media Characteristics, resulted from a collaborative effort with the National Aeronautics and Space Administration. Monitoring the status of data recorded on optical media is important because of its anticipated use for the long-term storage of valuable data. 
Distributed Systems. CSL continued to conduct research and participate in standards development for distributed computer systems as interconnected by ISDN. The development of a voluntary industry standard for Distributed Transaction Processing progressed, including a formal description of the protocol in Estelle, a simulation model, and a prototype implementation. Researchers also developed and demonstrated distributed applications using the protocol for accessing distributed multimedia information. Development of Application Profiles for ISDN is underway.

Research in distributed systems architectures resulted in the development of a unifying architecture called the Services Backplane which provides a common interface between applications and services. Work continued on a generalized information system including mixed media information (e.g., graphics, images, and text) via a Virtual Workstation Architecture. Researchers also participated in the definition and specification of the Application Software Interface being developed in the NIU-Forum.

Researchers are planning for CSL's participation in the national planned event for demonstrating standard ISDN as per the NIU-Forum objectives. Various profiles for imaging and data are being developed.

Automated Sponsored by the Bureau of the Census, CSL researchers developed a handRecognition. printed character database consisting of 2100 pages of bi-level, black and white, image data of hand-printed numerals and text stored in compressed form on CD-ROM (Compact Disk-Read Only Memory). The total image database, in uncompressed form, contains about 3 gigabytes of image data, with 273,000 numerals and 707,700 alphabetic characters. The hand-printing sample was obtained from a selection of field data collection staff of the Bureau of the Census, with a geographic sampling corresponding to the population of the United States.

Prior to the development of the database, no large public source of test data for the design and evaluation of character recognition technology was available. The costs of manually keying in data for computer processing in government and the financial sector of the U.S. economy are presently estimated at $\$ 20$ billion annually. Character recognition technology can significantly improve the productivity of these service sector activities. To date, 28 universities, industrial R\&D laboratories, and users of character recognition technology have acquired the database.

In another image recognition project, two researchers won the "Best Paper of 1991" Award from the American Society for Information Science (ASIS). The paper, "Retrieving Records from a Gigabyte of Text on a Minicomputer using Statistical Ranking," reported on the NIST Probabilistic Ranking Information Search Engine (PRISE) system which is a prototype retrieval system using very efficient algorithms to implement statistically based ranked retrieval of information. This type of ranking has Iong been known to be superior to traditional Boolean retrieval for the average user, but has received minimal commercial interest because of its reputed difficulty of implementation. 
Partially sponsored by DARPA, research in speech recognition technology proceeded in collaboration with academia and industry. The use of CD-ROM data storage media in the United States for the exchange of recorded speech databases ("corpora") within the speech research community continued to advance. In addition to $C D-R O M$ sets released for DARPA, further releases will be produced as reference material for use in speech research. CSL's work on the design and development of test procedures and other materials for the DARPA Spoken Language Systems Program and other Department of Defense speech research programs continued.

Integrated Services Digital Network (ISDN).
To ensure a strong user voice in the implementation of ISDN applications and to advance the standardization of ISDN technology in the United States, CSL continued to sponsor the North American ISDN Users' Forum (NIU-Forum). A Cooperative Research and Development Agreement (CRDA) with industry was established in FY 1991 to govern the management of the forum.

Three forum meetings were held in 1991 with about 300 vendor and user participants. Two new publications resulted. NIST Special Publication 500194, ISDN Conformance Testing, Layer 1 - Physical Layer, Part 1 - Basic Rate S/T Interface, User Side, describes a set of test specifications, developed by NlUForum members, which test conformance of Terminal Equipment (TEs) and Network Termination (NTs) to the ISDN Physical Layer at the S/T reference point, as defined in American National Standard (ANS) T1.605-1989. NIST Special Publication 500-195, North American ISDN Users' Forum Agreements on Integrated Services Digital Network, compiles the existing NIU-Forum agreements as of November 1990.

To advance ISDN as a global technology, CSL delegates represented the NIUForum ISDN Conformance Testing group (ICOT) at the Consultative Committee on International Telephony and Telegraphy (CCITT) Study Group XI meeting on ISDN conformance testing and Broadband ISDN protocols in September in Geneva, Switzerland. In the conformance testing area, the layer 2 abstract test suite developed by the ICOT was moved to "frozen" state, paving the way for final approval in April 1992 as a 1992 Recommendation named Q.921bis. This is the first time a CCITT study group has produced a Recommendation in less than two years. In the Protocol Implementation Conformance Statement (PICS) area, a CSL delegate chaired an ad hoc group meeting on layer 3 PICS which will be moved forward to the "awaiting review" state. Again, the NIU-Forum created a record of being the first to add a PICS to a CCITT recommendation. 


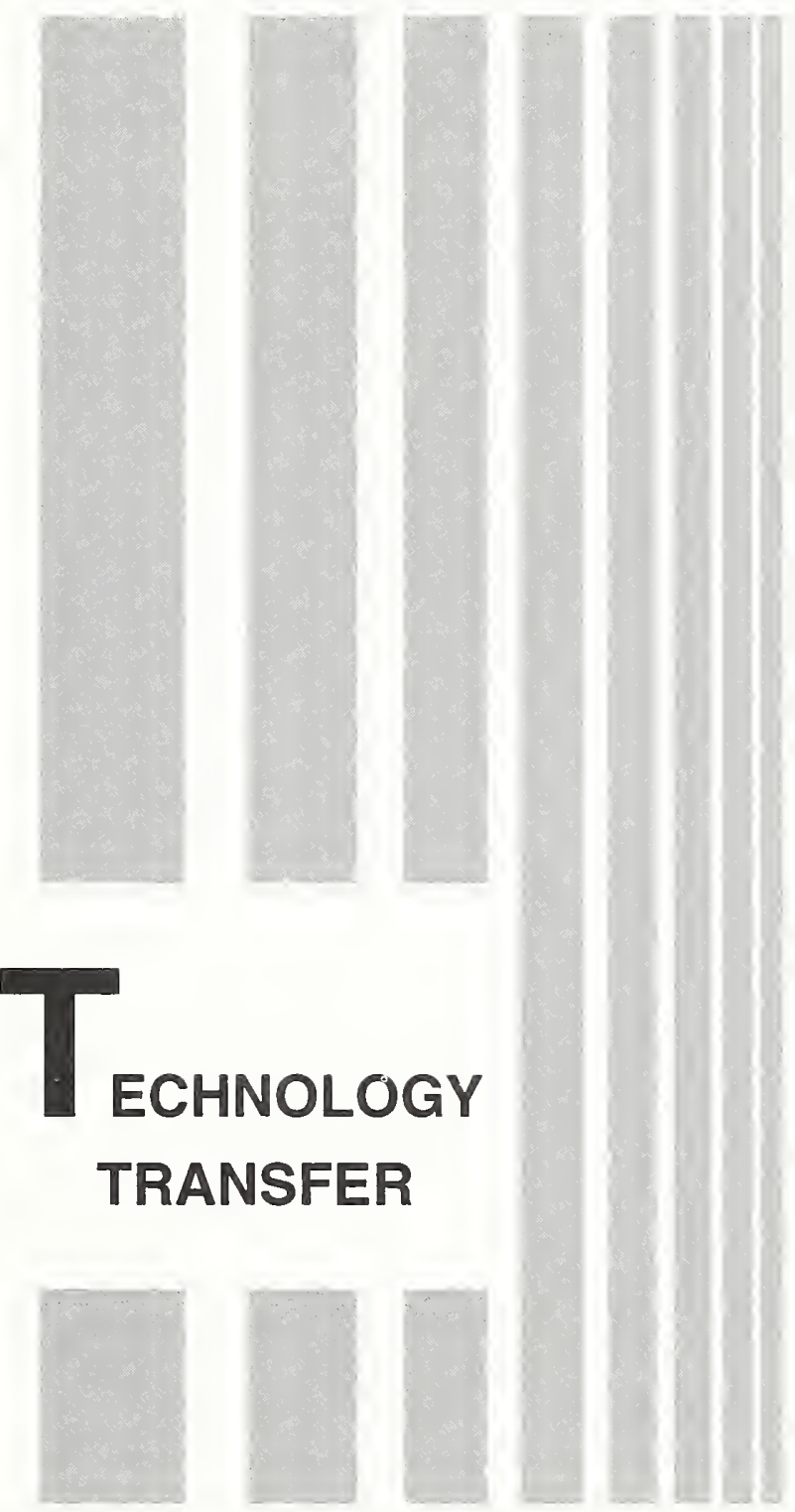




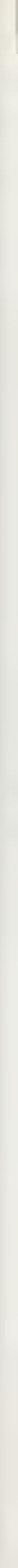




\section{PARTICIPATION IN VOLUNTARY STANDARDS ACTIVITIES}

CSL staff members participate in more than 85 national and international voluntary standards activities, including the following:

American National Standards Institute (ANSI):

Information Systems Standards Board (ISSB)

Information Technology Consultative Committee (ITCC)

USA Registration Authority Committee

\section{Accredited Standards Committee (ASC):}

Tl, Telecommunications

X3, Information Processing Systems

X9, Financial Services

$\mathrm{X} 12$, Electronic Data Interchange (EDI)

IT9, Physical Properties and Permanence of Imaging Media

Institute of Electrical and Electronics Engineers (IEEE):

IEEE Standards Board and Committees

IEEE Groups for:

Local Area Networks

Portable Operating System Interface (POSIX)

Graphical User Interface

Software Engineering

U.S. TAG for JTC 1 SC 7

U.S. TAG for JTC 1 SC 22 WG 15

Futurebus

International Organization for Standardization (ISO)/

International Electrotechnical Commission (IEC)

Joint Technical Committee 1 (JTC 1) on Information Technology

U.S. Technical Advisory Group (TAG) for ISO/IEC JTC 1 (JTC 1 TAG)

International Telegraph and Telephone Consultative Committee (CCITT)

Study Groups for:

Data Communications Networks

Switching and Signaling

\section{U.S. National Committee for CCITT:}

Study Group B

Study Group D 
International Organization for Standardization (ISO)

Technical Committees for:

Industrial Automation

Micrographics and Optical Memories for Document and Image

Recording, Storage and Use

ASC X3 Subgroups for:

BASIC

Computer Graphics

Credit/Identification Cards

Database

Data Communications

Data Interchange

Data Representation

Digital Magnetic Tape

I/O Interface

Information Resource Dictionary System

Information Technology Security Techniques

LISP

Open Distributed Processing

Open Systems Interconnection

Optical Digital Data Disks

Parallel Processing Constructs for High-Level Programming Languages

Picture Coding

Secretariat Management Committee (SMC)

Standards Planning and Requirements Committee (SPARC)

SPARC Database Systems Study Group

Text: Office and Publishing Systems

U.S. TAG for JTC 1 SC 21

U.S. TAG for JTC 1 SC 22

\section{ASC X9 Subgroups for:}

Data and Information Security

Public-Key Cryptography for Financial Institutions

Security for Financial Systems

Wholesale Banking

ASC X12 Subgroup for:

Security

X12/EDIFACT Alignment

ASC T1 Technical Subcommittee for:

Services, Architecture and Signaling 
JTC 1 TAG Subgroups for:

Advisory Committee

Applications Portability

EDI

Functional Standards

Procedures

Registration Authorities

ISO/IEC JTC 1 Subcommittees or Groups for:

Advisory Group

Applications Portability

Computer Graphics

Design and Documentation of Computer-Based Information Systems

Flexible Magnetic Media for Digital Data Interchange

Functional Standardization

Information Retrieval, Transfer and Management for OSI

Information Technology Security Techniques

Interconnection of Information Technology Equipment

Languages

Optical Disk Cartridges for Information Interchange

POSIX

Procedures

Registration Authorities

Representation of Data Elements

Telecommunications and Information Exchange Between Systems

Text and Office Systems

\section{European Computer Manufacturers Association (ECMA) Technical}

Committees or Task Groups for:

Lower Four OSI Layers and Local Area Networks

Magnetic Tapes

PCTE (Portable Common Tool Environment) TC33/TGEP

Reference Model for Software Environments TC33/TGRM

\section{European Workshop on Open Systems (EWOS)}

Expert Group on Common Application Environments (to be changed to OSE) EG-CAE

Association for Information and Image Management (AIIM)

\section{Canadian Committee on Geomatics}


Data Administration Management Association Standards and Procedures Subgroup

Federal Interagency Coordinating Committee on Digital Cartography

Federal Telecommunication Standards Committee

International Association for Identification

National Association of State Information Resource Executives (NASIRE)

NIST OSI Implementors Workshop (OIW)

North American ISDN Users' Forum (NIU-Forum)

U.S. Board on Geographic Names

CSL staff members hold key leadership positions in many of the above activities. 


\section{COLLABORATION WITH GOVERNMENT, INDUSTRY, AND ACADEMIA}

In 1991, CSL collaborated with the following agencies in government, industry, and academia, some of which supported CSL research through funding or the loan of equipment or software.

\section{Federal Agencies}

Department of Defense

Ada Joint Program Office

Air Force American Forces Information Service

Air Force Communications Command

Air Force, Scott Air Force Base, Illinois

Army Engineering Division, Huntsville, Alabama

Army, Fort Huachuca, Arizona

Army Information Systems Command

Army Sustaining Base Information Services

Computer-aided Acquisition and Logistics Support (CALS)

Corporate Information Management Office

Defense Advanced Research Projects Agency

Defense Communications Agency

Defense Logistics Agency

Joint Interoperability Test Center

Joint Tactical Command Control Communications Agency

National Security Agency

Naval Military Personnel Command

Navy Fleet Combat Direction System Support Activity

Navy Next Generation Computer Resources

Navy Space and Naval Warfare Systems Command Center

Navy Information Technical Acquisition Center

Office of the Secretary of Defense

Department of Commerce, Bureau of the Census

Department of Education

Department of Energy

Department of Health and Human Services

Department of the Interior

Department of Justice, Federal Bureau of Investigation

Department of State

Department of the Treasury, Financial Management Service

Department of the Treasury, Internal Revenue Service 
Federal Agencies (continued)

Department of Veterans Affairs

Environmental Protection Agency

Farmers Home Administration

Federal Emergency Management Agency

General Services Administration

National Aeronautics and Space Administration

National Archives and Records Administration

Nuclear Regulatory Agency

Office of Management and Budget

Securities and Exchange Commission

Social Security Administration

Tennessee Valley Authority

\section{Industry}

3Com

Bellcore

Data General

Digital Equipment Corporation

Environmental Sciences Research Institute

Geovision Corporation

Hewlett-Packard

Hughes Aircraft Company

Interactive Systems

International Business Machines

Network General

Novell

Prime Computer

RETIX

Servio Logic Corporation

Spatial Data Sciences

Sun Microsystems

Teledyne Brown

Textronix

TYDAC Corporation

Wollongong Group

Xerox

\section{Academia}

George Washington University

University of Toronto

Virginia Commonwealth University 


\section{CSL COOPERATIVE RESEARCH \&}

DEVELOPMENT AGREEMENTS (CRDAS)

As of January 6, 1992

RESEARCH PARTNER

Open Systems Environments

Sun Microsystems, Inc.

Digital Equipment Corporation

Eagen, William J.

Minderaft, Inc.

X/Open Ltd.

International Business

Machines Corporation

Open Software Foundation

Oracle Federal Division
PROJECT

Application Portability Profile (APP)

Application Portability Profile (APP)

Application Portability Profile (APP)

Application Portability Profile (APP)

Application Portability Profile (APP)

Application Portability Profile (APP)

Graphical User Interface Mathematical Methods Information Systems Life Cycle Cost Savings

Computer Systems Security

\section{Computer Security}

Bellcore

(ISDN)

Integrated Services Digital Network (ISDN)

UNITEL Communications, Inc.

NIU-Forum (North American ISDN Users' Forum)

U.S. Sprint NIU-Forum

Siemens Stromberg-Carlson Ameritech NIU-Forum Eastman Kodak Company NIU-Forum First National Bank of Chicago, The NIU-Forum AT\&T Bell Labs NIU-Forum Bell Communications Research NIU-Forum Pacific Bell NIU-Forum Aeronautical Radio, Inc. (ARINC) NIU-Forum NYNEX NIU-Forum NIU-Forum American Management Systems, Inc. NIU-Forum TASC (The Analytic Sciences Corp.) NIU-Forum Verilink Corporation NIU-Forum CIMI Corporation NIU-Forum 
RESEARCH PARTNER

American Computer \& Electronics

Corporation

U.S. West

Vanguard Research

Boeing Computer Support

Services, Inc.

Southwestern Bell

U.S. Air Force (Technology

Integration Center)

Raynor Associates, Inc.

Motorola, Inc.

Electronic Data Systems Corporation Idacom Electronics, Ltd.

General DataComm, Inc.

National Aeronautics and Space

Administration (NASA)

Hayes Microcomputer Products, Inc.

Mitel Corporation

Memorex Telex Corporation

Department of Defense (National Security Agency)

Bell Atlantic

Defense Communication Agency

International Business Machines Corporation

University of Michigan

Teleos Communications, Inc.

Rome Research Corporation

Baxter Healthcare Corporation

Schindler Elevator Corporation

Johnson \& Johnson Hospital

Services, Inc.

Northern Telecom

DGM\&S, Inc.

Bell Communications Research

Loral Aerospace

COMSAT Corporation

Datacom, Inc.

Tekelec, Inc.
PROJECT

NIU-Forum

NIU-Forum

NIU-Forum

NIU-Forum

NIU-Forum

NIU-Forum

NIU-Forum

NIU-Forum

NIU-Forum

NIU-Forum

NIU-Forum

NIU-Forum

NIU-Forum

NIU-Forum

NIU-Forum

NIU-Forum

NIU-Forum

NIU-Forum

NIU-Forum

NIU-Forum

NIU-Forum

NIU-Forum

NIU-Forum

NIU-Forum

NIU-Forum

NIU-Forum

Broadband ISDN Standards and Technology

Integrated Services

Digital Network

Integrated OSI, ISDN, and

Security Program

Test and Demonstrate ISDN

Protocols and Services

EDI and ISDN

X-25 Standards Tests 
RESEARCH PARTNER

Multimedia Technology

Interactive Multimedia Association

Open Systems and Networks

Aeronautical Radio, Inc.

(ARINC)

Corporation for Open Systems

Software Standards Validation

Washington Software Technologies, Inc.

Speech Research

NYNEX Corporation

Parallel Processing Research

Convex Computer Corporation
PROJECT

Multimedia Forum

Network Management Testbed

GOSIP

Basic Test Suite

N-TIMET Database

MULTIKRON Project 


\section{GUEST RESEARCHERS FY 1991}

Guest Scientists and Research Associates

Organizations represented included:

\section{Bellcore}

Environmental Protection Agency

Institute of Geology, Beijing, China

Institut National Des Telecommunications, France

International Business Machines

International Computers Ltd., United Kingdom

National Science Foundation

Northeast University of Technology

Armament Development Authority, Rafael, Haifa, Israel

Space Science \& Tech Center, Chinese Academy of Science

Sun Microsystems

Swedish Telecom \& Scholarship

Telecommunications Laboratory, Ministry of Communications, Taiwan

University of Bordeaux, France

University of Twente, Netherlands

Washington Software Technologies Inc., Annandale, VA

University of Maryland, College Park, MD

University of Maryland, Baltimore, MD

University of Pittsburgh 


\section{NIST PUBLICATIONS}

\section{NIST COMPUTER SYSTEMS \\ TECHNOLOGY SERIES \\ FY 1989 - FY 1991}

NIST SPEC. PUB. TITLE

500-159 Data Administration: Management and Practice - Proceedings of the First DAMA Symposium

Judith J. Newton and Frankie E. Spielman, Editors

SN 003-003-02901-7 \$7.00 Oct 1988

500-160 Report of the Invitational Workshop on Integrity Policy in Computer Information Systems (WIPCIS)

Stuart W. Katzke and Zella R. Ruthberg, Editors

SN 003-003-02904-1 \$11.00 Jan 1989

500-161 Software Configuration Management: An Overview

By Wilma Osborne

SN 003-003-02927-1 \$2.00 Mar 1989

500-162 Stable Implementation Agreements for Open Systems Interconnection Protocols -

Version 2, Edition 1

Tim Boland, Workshop Chairman

SN 003-003-02921-1 \$26.00 Dec 1988

500-163 Government Open Systems Interconnection Users' Guide

By Tim Boland

PB 90-111212 \$23.00 Aug 1989

500-164 Electronic Publishing: Guide to Selection

By Lynne Rosenthal

SN 003-003-02938-6 \$2.50 Jun 1989

500-165 Software Verification and Validation: Its Role in Computer Assurance and Its Relationship with Software Product Management Standards

By Dolores Wallace and Roger Fujii

SN 003-003-02959-9 \$2.25 Sep 1989

500-166 Computer Viruses and Related Threats: A Management Guide By John P. Wack and Lisa J. Carnahan

SN 003-003-02955-6 \$2.50 Aug 1989

SN numbers - stocked by GPO

PB numbers - stocked by NTIS 
NIST SPEC. PUB. TITLE

500-167 Information Management Directions: The Integration Challenge By Elizabeth N. Fong and Alan H. Goldfine SN 003-003-02973-4 \$9.00 Sep 1989

500-168 Report of the Invitational Workshop on Data Integrity By Zella G. Ruthberg and William T. Polk

SN 003-003-02966-1 \$20.00 Sep 1989

500-169 Executive Guide to the Protection of Information Resources By Cheryl Helsing, Marianne Swanson, and Mary Anne Todd SN 003-003-02969-6 \$1.50 Oct 1989

500-170 Management Guide to the Protection of Information Resources By Cheryl Helsing, Marianne Swanson, and Mary Anne Todd SN 003-003-02968-8 \$1.75 Oct 1989

500-171 Computer Users' Guide to the Protection of Information Resources By Cheryl Helsing, Marianne Swanson, and Mary Anne Todd SN 003-003-02970-0 \$1.00 Oct 1989

500-172 Computer Security Training Guidelines By Mary Anne Todd and Constance Guitian SN 003-003-02975-1 \$2.50 Nov 1989

500-173 Guide to Data Administration By Bruce K. Rosen and Margaret H. Law SN 003-003-02967-0 \$4.25 Oct 1989

500-174 Guide for Selecting Automated Risk Analysis Tools By Irene E. Gilbert

SN 003-003-02971-8 \$2.00 Oct 1989

500-175 Management of Networks Based on Open Systems Interconnection (OSI) Standards: Functional Requirements and Analysis

By Robert Aronoff, Michael Chernick. Karen Hsing, Kevin Mills, and Daniel Stokesberry SN 003-003-02986-6 \$7.00 Nov 1989

500-176 Introduction to Heterogeneous Computing Environments By John Barkley and Karen Olsen PB 90-154774 \$11.00 Nov 1989

SN numbers - stocked by GPO

PB numbers - stocked by NTIS 
NIST SPEC. PUB. TITLE

500-177 Stable Implementation Agreements for Open Systems Interconnection Protocols, Version 3, Edition 1, Dec 1989

Tim Boland, Workshop Chairman

SN 003-003-02995-5 \$31.00 Dec 1989

500-178 Proceedings of the Hypertext Standardization Workshop, January 16-18, 1990 By J. Moline, D. Benigni, and J. Baronas

SN 003-003-02998-0 \$14.00 Mar 1990

500-179 Object Database Management Systems: Concepts and Features

By C. Dabrowski, E. Fong, and D. Yang

SN 003-003-03007-4 \$3.75 Apr 1990

500-180 Guide to Software Acceptance

By Dolores Wallace and J. Cherniavsky

SN 003-003-03008-2 \$2.50 Apr 1990

500-181 PHIGS Validation Tests (Version 1.0): Design Issues

By John Cugini, Mary T. Gunn, and Lynne S. Rosenthal

SN 003-003-03028-7 \$1.75 Jul 1990

500-182 Guidelines for the Evaluation of Message Handling Systems Implementation

By Steve Trus, Curtis Royster, and Paul Markovitz

PB 90-269598 \$23.00 Aug 1990

500-183 Stable Implementation Agreements for Open System Interconnection Protocols, Version 4, Edition 1, December 1990

Tim Boland, Workshop Chairman

SN003-015-00000-4 \$50.00 Dec 1990

500-184 Functional Benchmarks for Fourth Generation Languages

By Martha M. Gray and Gary E. Fisher

SN003-003-03071-6 \$3.25 Mar 1991

500-185 Guide to Design, Implementation and Management of Distributed Databases By Elizabeth N. Fong, Charles L. Sheppard, and Kathryn A. Harvill SN003-003-03076-7 \$3.50 Feb 1991

500-186 Issues in Transparent File Access

By Karen Olsen and John Barkley

SN003-003-03079-1 \$4.75 Apr 1991

SN numbers - stocked by GPO

PB numbers - stocked by NTIS 
NIST SPEC. PUB. TITLE

500-187 Application Portability Profile (APP) The U.S. Government's Open System Environment Profile OSE/1 Version 1.0

PB91-201004 \$19.00 Apr 1991

500-188 Guide to Expert System Building Tools for Microcomputers

By Christopher E. Dabrowski and Elizabeth N. Fong

SN003-003-03088-1 \$8.50 Jul 1991

500-189 Security in ISDN

By William E. Burr

SN003-003-03112-7 $\quad \$ 4.25 \quad$ Sep 1991

500-190 Proceedings of the Workshop on High Integrity Software; Gaithersburg, MD; Jan. 22-23, 1991

By Dolores R. Wallace, D. Richard Kuhn, and John C. Cherniavsky

SN003-003-03108-9 \$5.50 Aug 1991

500-191 Test Methods for Optical Disk Media Characteristics (for 356 mm Ruggedized Magneto-optic Media)

By Fernando L. Podio PB92-116409 \$19.00 Sep 1991

500-192 Government Open Systems Interconnection Profile Users' Guide, Version 2 By Tim Boland SN003-003-03119-4 \$9.50 Oct 1991

500-193 Software Reengineering: A Case Study and Lessons Learned By Mary K. Ruhl and Mary T. Gunn SN003-003-03100-3 \$2.25 Sep 1991

500-194 ISDN Conformance Testing, Layer 1-Physical Layer, Part 1-Basic Rate S/T Interface, User Side

Shukri A. Wakid and Kathleen M. Roberts, Editors

PB92-102201 \$19.00 Sep 1991

500-195 North American ISDN Users' Forum Agreements on Integrated Services Digital Network

Shukri A. Wakid and Kathleen M. Roberts, Editors

PB92-102219

$\$ 26.00$ Sep 1991

SN numbers - stocked by GPO

PB numbers - stocked by NTIS 
NIST SPEC. PUB. TITLE

500-196 Guidelines for the Evaluation of File Transfer, Access and Management

Implementations

By Paul Markovitz, Steve Trus, and Curtis Royster

SN003-003-03120-8 \$5.50 Oct 1991

500-197 Guide to Schema and Schema Extensibility

By Bruce K. Rosen and Isabella des Fontaines

SN003-003-03126-7 \$2.25 Nov 1991

500-198 Monitoring and Reporting Techniques for Error Rate and Error Distribution in Optical Disk Systems

By Fernando L. Podio

SN003-003-03125-9 \$5.00 Oct 1991

500-199 The 3480 Type Tape Cartridge: Potential Data Storage Risks, and Care and Handling Procedures to Minimize Risks

By Mark P. Williamson

SN003-003-03127-5 \$3.50 Nov 1991

SN numbers - stocked by GPO

PB numbers - stocked by NTIS 


\section{NIST COMPUTER SECURITY SERIES FY 1991}

NIST SPEC. PUB. TITLE

800-1 Computer Security in the 1980s: Selected Bibliography Rein Turn, Compiler, and Lawrence E. Bassham, Editor SN003-003-03060-1 \$11.00 Dec 1990

800-2 Public-Key Cryptography

By James Nechvatal

SN003-003-03078-3 \$9.00 Apr 1991

800-3 Establishing a Computer Security Incident Response Capability (CSIRC) By John P. Wack

SN003-003-03121-6 \$3.00 Nov 1991

SN numbers - stocked by GPO

PB numbers - stocked by NTIS 


\section{OTHER NIST PUBLICATIONS FY 1989 - FY 1991}

PUB. NUMBER TITLE

NISTIR 88-3896 Proceedings of the Federal Information Processing Standards Workshop on Information Resource Dictionary System (IRDS) Applications Alan Goldfine, Editor

December $1988 \quad$ PB89-136329 \$21.95

NISTIR 88-4001 Wavefront Matrix Multiplication on a Distributed-Memory Multiprocessor James Nechvatal, Editor

January 1989 PB89-151807 \$15.95

NISTIR 88-4017 Standards for the Interchange of Large Format Tiled Raster Documents By Frankie Spielman

December $1988 \quad$ PB89-148415 \$15.95

NISTIR 88-4022 Issues in Interpreting The Export Administration Regulations,

"Processing Data Rate"

By Vivian Lawrence

February 1989

Available from Advanced Systems Division

NISTIR 89-4023 Small Computer System Interface (SCSI) Command System: Software Support for Control of Small Computer System Interface Devices By John Gorczyca and Eduardo Villagran

January $1989 \quad$ PB89-151815 \$21.95

NISTIR 89-4053 Architecturally-Focused Benchmarks with a Communication Example By G.E. Lyon, R. D. Snelick

$\begin{array}{lll}\text { March } 1989 & \text { PB89-216477 } \$ 13.95\end{array}$

NISTIR 89-4063 Hybrid Structures for Simple Computer Performance Estimates By Gordon Lyon

March $1989 \quad$ PB89-189161 \$13.95

NISTIR 89-4128 Processing Rate Sensitivities of a Heterogeneous Multiprocessor By G.E. Lyon

August $1989 \quad$ PB89-229017 \$13.95

SN numbers - stocked by GPO

PB numbers - stocked by NTIS 
PUB. NUMBER TITLE

NISTIR 89-4139 A Detailed Description of the Knowledge-Based System for Physical Database Design (2 volumes)

By Christopher E. Dabrowski

August $1989 \quad$ PB89-228993 (vol. 1) $\$ 17.00$

PB89-229003 (vol. 2) $\$ 23.00$

NISTIR 89-4140 Working Implementation Agreements for Open System Interconnection Protocols Tim Boland, Editor

September $1989 \quad$ PB89-235931 \$36.95

NISTIR 89-4158 OSINET General Agreements and Information Document

By Gerard F. Mulvenna

August 1989

Restricted to NIST use only

NISTIR 89-4160 Trial of Open System Interconnection (OSI) Protocols Over Integrated Services

Digital Network (ISDN)

By Carol A. Edgar

August $1989 \quad$ PB89-235576 \$13.95

NISTIR 89-4169 Use of the IRDS Standard in CALS

By David K. Jefferson and Cita M. Furlani

September 1989 PB91-132209 \$15.00

NISTIR 89-4198 Working Implementation Agreements for Open Systems Interconnection Protocols Tim Boland, Editor

December 1989 PB90-146440 \$44.00

NISTIR 89-4199 Graphics Application Programmer's Interface Standards and CALS By Sharon Kemmerer and Mark Skall

October $1989 \quad$ PB90-133091 \$15.00

NISTIR 90-4228 Prototyping SP4 A Secure Data Network System Transport Protocol Interoperability Demonstration Project

By C. Dinkel, N. Nazario, and R. Rosenthal

January $1990 \quad$ PB90-159609 \$15.00

NISTIR 90-4240 Architectures for Future Multigigabit Lightwave Networks

By W. Burr

February $1990 \quad$ PB90-198953 \$19.00

SN numbers - stocked by GPO

PB numbers - stocked by NTIS 
PUB. NUMBER TITLE

NISTIR 90-4247 Working Implementation Agreements for Open Systems Interconnection Protocols Tim Boland, Editor

March $1990 \quad$ PB90-197948 \$30.00

NISTIR 90-4250 Secure Data Network System (SDNS) Network, Transport, and Message Security Protocols

By C. Dinkel

February $1990 \quad$ PB90-198946 \$17.00

NISTIR 90-4259 Secure Data Network System (SDNS) Access Control Documents

By C. Dinkel

February $1990 \quad$ PB90-188061 \$23.00

NISTIR 90-4262 Secure Data Network System (SDNS) Key Management Documents

By C. Dinkel

February $1990 \quad$ PB90-188079 \$17.00

NISTIR 90-4267 A Conformance Test for FDDI Medium Access Control (MAC)

By Z. Liu and W. Burr

July $1990 \quad$ PB90-265323 \$23.00

NISTIR 90-4275 Workloads, Observables, Benchmarks and Instrumentation

By G. Lyon and R. Snelick

April $1990 \quad$ PB90-207770 \$15.00

NISTIR 90-4292 Data Administration: Standards and Techniques, Proceedings of the Second Annual DAMA Symposium

By J. Newton and F. Spielman

April $1990 \quad$ PB90-204512 \$23.00

NISTIR 4302 Working Implementation Agreements for Open Systems Interconnection Protocols Tim Boland, Editor

June $1990 \quad$ PB91-120113 \$45.00

NISTIR 4315 A Collection of Technical Studies Completed for the Computer-Aided Acquisition and Logistic Support (CALS) Program Fiscal Year 1988, Volume 1 of 3, Security and Data Management April 1990 PB91-178889 \$31.00

NISTIR 4316 A Collection of Technical Studies Completed for the CALS Program Fiscal Year 1988, Volume 2 of 3, Graphics, CGM MIL SPEC

$\begin{array}{lll}\text { April } 1990 & \text { PB91-178887 } \$ 45.00\end{array}$

SN numbers - stocked by GPO

PB numbers - stocked by NTIS 
PUB. NUMBER TITLE

NISTIR 4317 A Collection of Technical Studies Completed for the CALS Program Fiscal Year 1988, Volume 3 of 3, CGM Registration

April $1990 \quad$ PB91-178905 \$23.00

NISTIR 4325 U.S. Department of Energy Risk Assessment Methodology

E. Roback, NIST Coordinator

May $1990 \quad$ PB90-244484 \$23.00

NISTIR 4329 Graphics Standards in the Computer-Aided Acquisition and Logistic Support (CALS) Program, FY 1989, Volume 1: Test Requirements Document, Extended CGM (CGEM)

D. Benigni, Editor

July $1990 \quad$ PB90-257759 \$38.00

NISTIR 4330 Graphics Standards in the Computer-Aided Acquisition and Logistic Support (CALS) Program, FY 1989, Volume 2: MIL-D-28003 Revisions, CGM Registration D. Benigni, Editor May $1990 \quad$ PB90-228016 \$38.00

NISTIR 4331 Emulation Through Time Dilation By J. Antonishek and R. Snelick

May $1990 \quad$ PB90-228024 \$15.00

NISTIR 4349 User's Guide for the PHIGS Validation Tests (Version 1.0) By J. Cugini, M. Gunn, and L. Rosenthal

August $1990 \quad$ PB90-265216 \$23.00

NISTIR 4359 Domestic Disaster Recovery Plan for PCs, OIS, and Small VS Systems E. Roback, NIST Coordinator August $1990 \quad$ PB90-265240 \$15.00

NISTIR 4362 Security Labels for Open Systems An Invitational Workshop By N. Nazario

June $1990 \quad$ PB90-247446 \$30.00

NISTIR 4374 The Naming Forum: Proceedings of the IRDS Workshop on Data Entity Naming Conventions

By J. Newton

July $1990 \quad$ PB90-250119 \$23.00

NISTIR 4377 A Framework for Developing a CALS Data Dictionary

By D. Jefferson

July $1990 \quad$ PB90-257585 \$15.00

SN numbers - stocked by GPO

PB numbers - stocked by NTIS 
PUB. NUMBER TITLE

NISTIR 4378 Automated Information System Security Accreditation Guidelines

E. Roback. NIST Coordinator

August $1990 \quad$ PB90-264102 $\$ 15.00$

NISTIR 4382 Working Implementation Agreements for Open Systems Interconnection Protocols Tim Boland, Editor

September $1990 \quad$ PB90-259763 $\$ 45.00$

NISTIR 4384 Design Issues for Conformance Testing of the PHIGS Standard

By J. Cugini and L. Rosenthal

August $1990 \quad$ PB90-264094 \$15.00

NISTIR 4387 U.S. Department of Justice Simplified Risk Analysis Guidelines (SRAG)

E. Roback, NIST Coordinator

August $1990 \quad$ PB90-265257 $\$ 17.00$

NISTIR 4404 Dynamic Characteristics of Hypertext

By R. Furuta and P. David Stotts

August $1990 \quad$ PB91-107276 \$15.00

NISTIR 44091989 Computer Security and Privacy Plans (CSPP) Review Project: A First-Year Federal Response to the Computer Security Act of 1987 (Final Report)

Dennis M. Gilbert, Report Coordinator

September $1990 \quad$ PB91-107504 \$23.00

NISTIR 4418 State Occupancy Information for Performance Comparisons

By Gordon Lyon

October $1990 \quad$ PB91-112879 \$15.00

NISTIR 4432 Status of PDES-Related Activities (Standards \& Testing)

By Cita Furlani, Joan Wellington, and Sharon Kemmerer

October $1990 \quad$ PB91-112888 $\$ 15.00$

NISTIR 4435 FTAM Interoperability Test

By Carol Edgar

August $1990 \quad$ PB91-107565 \$23.00

NISTIR 4448 Working Implementation Agreements for Open Systems Interconnection

Protocols-November 1990

Tim Boland, Editor

November $1990 \quad$ PB91-144444 \$60.00

SN numbers - stocked by GPO

PB numbers - stocked by NTIS 
PUB. NUMBER TITLE

NISTIR 4451 U.S. Department of Commerce Methodology for Certifying Sensitive Computer Applications

Edward Roback, NIST Coordinator

November $1990 \quad$ PB91-120162 \$17.00

NISTIR 4452 Message Handling Systems Interoperability Tests

By Carol Edgar

October $1990 \quad$ PB91-112789 \$17.00

NISTIR 4453 SRI International Improving the Security of Your UNLX System

Edward Roback, NIST Coordinator

November $1990 \quad$ PB91-120121 \$17.00

NISTIR 4484 Multimedia Courseware in an Open Systems Environment: A Federal Strategy By Judi Moline, Allen L. Hankinson, and Lawrence A. Welsch

December $1990 \quad$ PB91-143362 \$17.00

NISTIR 4488 Proceedings of the Object-Oriented Database Task Group Workshop; Tuesday, October 23, 1990. Chateau Laurier Hotel, Ottawa, Canada

Elizabeth N. Fong, Editor

January $1991 \quad$ PB91-157198 \$23.00

NISTIR 4494 SQL3 Support for CALS Applications

By Leonard Gallagher

December $1990 \quad$ PB91-167262 \$15.00

NISTIR 4500 Validated Processor List

Judy B. Kailey, Editor

January $1991 \quad$ PB91-187716 \$17.00

NISTIR 4503 Proceedings of the Object-Oriented Database Task Group Workshop. Tuesday. May 22, 1990, Atlantic City, $N J$

By Elizabeth N. Fong

February $1991 \quad$ PB91-159723 \$39.00

NISTIR 4507 Working Implementation Agreements for Open Systems Interconnection Protocols (December 1990)

Tim Boland, Editor

March $1991 \quad$ PB92-126523 \$43.00

NISTIR 4518 National Aeronautics and Space Administration's (NASA) Automated Information Security Handbook

Edward Roback, NIST Coordinator

March $1991 \quad$ PB91-187781 \$23.00

SN numbers - stocked by GPO

PB numbers - stocked by NTIS 
PUB. NUMBER TITLE

NISTIR 4524 Raster Graphics Conformance Testing

By Frankie E. Spielman

February $1991 \quad$ PB91-167296 \$17.00

NISTIR 4530 Validation of an OSI Transport Class 4 Simulator By Okhee Kim, Sharon Heatley, and Bob Bishop

May $1991 \quad$ PB91-187724 $\$ 15.00$

NISTIR 4545 Computer Security: Selected Articles

Marianne Swanson and Elizabeth B. Lennon, Editors

April $1991 \quad$ PB91-187740 \$15.00

NISTIR 4547 A Standardized General Markup Language Encoding of the Office Document Architecture Document Application Profile

By Ronald B. Wilson

April $1991 \quad$ PB91-184812 \$15.00

NISTIR 4557 Programming Languages and Database Language SQL Validated Processor List Including GOSIP Conformance Testing Registers Judy B. Kailey, Editor

April $1991 \quad$ PB91-187716 \$17.00

NISTIR 4560 Government Document Processing Requirements Report

By Roger F. Sies

April $1991 \quad$ PB91-187773 $\$ 15.00$

NISTIR 4567 Titled Raster Graphics and MIL-R-28002A: A Tutorial and Implementation Guide By Frankie E. Spielman and Louis H. Sharpe, II

April $1991 \quad$ PB91-187708 \$23.00

NISTIR 4579 NIST Support for the Computer-Aided Acquisition and Logistic Support (CALS)

Program in the Area of Graphics Standards, Calendar Year 1990

By Daniel Benigni

May $1991 \quad$ PB91-194506 \$39.00

NISTIR 4594 GOSIP Conformance and Interoperation Testing and Registration Nightingale, J.S.

March $1991 \quad$ PB92-108943 \$26.00

NISTIR 4607 Network Registration Service User's Guide

By Carol Edgar

June 1991

Available from OSINET Corporation

SN numbers - stocked by GPO

PB numbers - stocked by NTIS 
PUB. NUMBER TITLE

NISTIR 4608 Electronic Data Interchange in Message Handling Systems By Paul Markovitz

June $1991 \quad$ PB91-216622 \$15.00

NISTIR 4609 NIST Support of the CALS Program: 1990 Synopsis

By Sharon J. Kemmerer

June $1991 \quad$ PB91-193821 \$15.00

NISTIR 4614 Standard Security Label for GOSIP, An Invitational Workshop

By Noel Nazario

June $1991 \quad$ PB91-216671 \$23.00

NISTIR 4630 Performance Evaluation of Hypercube Applications: Using a Global Clock and Time Dilation

By Robert D. Snelick

July $1991 \quad$ PB91-222588 \$15.00

NISTIR 4636 U.S. Dept. of Health and Human Services Automated Information Systems

Security Program Handbook

Edward Roback, NIST Coordinator

July $1991 \quad$ PB91-222638 \$23.00

NISTIR 4637 Computer Implementation of a Discrete Set Algebra

By Leonard Gallagher

July $1991 \quad$ PB91-231571 \$15.00

NISTIR 4651 Government Network Management Profile (GNMP): Public Review Version

By R. Aronoff, K. Brady, M. Chernick, J. Fox, K. Hsing, K. Mills, and F. Nielsen

NISTIR 4654 Development of an Optical Disk System for the Automated Retrieval of EASEAR Records

By Natalie Willman

August $1991 \quad$ PB92-108943 \$26.00

NISTIR 4655 User's Manual for the NIST TTCN Translator Version 3.0

By David H. Su

August 1991

SN numbers - stocked by GPO

PB numbers - stocked by NTIS 
PUB. NUMBER TITLE

NISTIR 4656 Proceedings of the Forum on Standards for High Integrity Software By Dolores R. Wallace, Michael Brown, and Archibald McKinlay VI September $1991 \quad$ PB92-112267 $\$ 19.00$

NISTIR 4659 Glossary of Computer Security Terminology Edward Roback, NIST Coordinator September $1991 \quad$ PB92-1 $12259 \quad \$ 26.00$

NISTIR 4667 Computer Security Bulletin Board System User's Guide By Mark Skandera and Marianne Swanson September $1991 \quad$ PB92-112390 \$17.00

NISTIR 4668 The OSINET Testing and Registration [T\&R] Service Functional Specification By Carol Edgar and Alper Kerman Available from OSINET Corporation

NISTIR 4688 Prototyping the IRDS: An Airport Application By Anthony P. Sani (Henry Tom, NIST Coordinator) October $1991 \quad$ PB92-112580 \$26.00

NISTIR 4739 Validated Processor List Judy B. Kailey, Editor January $1992 \quad$ PB91-937302 \$19.00 Available by subscription at $\$ 68.00$ per year.

$\mathrm{SN}$ numbers - stocked by GPO

PB numbers - stocked by NTIS 


\section{CSL BULLETINS}

\section{JUNE 1990 THROUGH NOVEMBER 1991}

Roback, Edward; Data Encryption Standard. NCSL Bulletin. June 1990.

Flahavin, Ellen; Guidance to Federal Agencies On The Use Of Trusted Systems Technology. NCSL Bulletin. July 1990.

Wack, John; Kurzban, Stanley A.; Computer Virus Attacks. NCSL Bulletin. August 1990.

McCrea, Samuel; Bibliography of Computer Security Glossaries. NCSL Bulletin. September 1990.

Gilbert, Dennis; Review of Federal Agency Computer Security and Privacy Plans (CSPP) A Summary Report. NCSL Bulletin. October 1990.

Roback, Edward; Computer Security Roles of NIST and NSA. CSL Bulletin. February 1991.

Roback, Edward; FIPS 140 - A Standard in Transition, CSL Bulletin. April 1991.

Radack, Shirley; The Gosip Testing Program. CSL Bulletin. May 1991.

Saltman, Roy; Security Issues in the Use of Electronic Data Interchange. CSL Bulletin. June 1991.

Boland, Tim; File Transfer, Access and Management. CSL Bulletin. July 1991.

Lennon, Elizabeth; The NIST POSIX Testing Program. CSL Bulletin. October 1991.

Dray, James; Advanced Authentication Technology. CSL Bulletin. November 1991. 


\section{PAPERS}

\section{OCTOBER 1990 THROUGH DECEMBER 1991}

Wong, William; Summary of the 4th Workshop on Integrated Software Engineering Environments (ISEE). October 29, 1990.

Favreau, Jean-Philippe; Mills, Kevin L; Nightingale, Stephen; The U.S. Gosip Testing Program. Proceedings of the 6th International Conference on the Application of Standards of Open Systems. October 1990.

Mills, Kevin L.; Standards and Trade: What's the Connection? Proceedings of the 6th International Conference on the Application of Standards for Open Systems. October 1990.

Steinauer, Dennis; Latest Research on Minimizing Security Risks for PCs and Lans. 10th Annual Conference on Control, Audit and Security of Information Systems. October 1990.

Wilson, Charles L.; A New Self-Organizing Neural Network Architecture for Parallel Multi-Map Pattern Recognition - FAUST. Progress in Neural Networks. October 1990.

Lennon, Elizabeth B.; North American Integrated Services Digital Network (ISDN) Users Forum (NIU-FORUM). NIST Journal of Research. November/December 1990.

Katzke, Stuart W.; A Framework for Computer Security Risk Management. Proceedings for the Society for Risk Analysis. November 29, 1990.

Zelkowitz, Marvin V.; A Functional Correctness Model of Program Verification, IEEE Computer 23(11). November, 1990.

Furuta, Richard; Stotts, P. David; A Functional Meta-Structure for Hypertext Models and Systems. Electronic Publishing, December 1990.

Kuhn, Richard D.; Dray, James F.; Formal Specifications and Verification of Control Software for Cryptographic Equipment. Sixth Annual Computer Security Applications Conference. December 1990.

Zelkowitz, Martin V.; Sergio Cardenas-Garcia; and Pablo Straub; Evaluation of Software Design Guality. Third International Workshop on Software Quality Improvement, Tokyo, Japan. January 1991.

Fong, Elizabeth N.; (editor); Workshop on Objects in Data Management. Proceedings of the Third Joint Meeting in Anaheim. California. January 14-15, 1991.

Branstad, Dennis K.; Katzke, Stuart W.: NIST Leads Development of Computer Security Standards. SIGNAL. January 1991. 
Su, David H.; Collica, Leslie A.; ISDN Conformance Testing. Proceedings of the IEEE, Vol. 79. No. 2. February 1991.

Wakid, Shukri A.; Roberts, Kamie; Application Profile for ISDN. Proceedings of the IEEE, Vol. 79, No. 2. February 1991.

Fong. Elizabeth N.; The DARPA/NIST Persistent Object Base Testbed. Position paper for the Open Object Oriented Data Base (OODB) Workshop. Dallas. Texas. March 13-15, 1991.

Nightingale, J.S.; Mills, K.L.; Favreau, J.P.; The U.S. Gosip Testing Program Progress and Outlook. Worldwide Recognition of OSI Test Results Workshop Proceedings. May 1991.

Zelkowitz, Marvin V.; Sergio Cardenas-Garcia; The Role for Executable Specifications in System Maintenance. Information Sciences Journal 57, 1991.

Barker, Elaine B.; Specialized Services and Security. Chapter for Handbook of Information Security Management.

Harman, D.; User-Friendly Systems Instead of User-Friendly Front-Ends. Journal of American Society for Information Science.

Aronoff, Richard; Chernick, C.M.; Stokesberry, D.P.; OSI Systems Management Standards. Proceedings of IEEE MILCOM '91 Conference.

Nielsen, Fran; An Overview of the Government Network Management Profile (GNMP). Proceedings of IEEE MILCOM '91 Conference.

Moline, Judi: Using Standards to Facilitate Access and Reuse of Information. Proceedings of ICHIM '91.

Harman, Donna K.; Closing the Vast Technology Transfer Gap in Text Retrieval. Proceedings of AAA1-91 Natural Language Text Retrieval Workshop.

Podio, Fernando L.: Status of Emerging Standards for Removable Computer Storage Media and Related Contributions of NIST. Proceedings of the Conference on Mass Storage Systems and Technologies for Space and Earth Science Applications. July 1991.

Wong, William; Summary of the 5th Workshop on Integrated Software Engineering Environments (ISEE). July 26, 1991.

Saltman, Roy; A Report to the State of Delaware Office of Information Systems on the Subject of Electronic Voting Machines - Source Book on Elections of the Computer Professionals for Social Responsibilities. 
Welsch, Larry A.; A Proposed International Interactive Courseware Standard. ITEC Publication.

Tom, Henry; GIS Standards: The Integration of Spatial Data Standards and Information Technology (IT) Standards. GIS World Magazine.

Wallace, D.R.; Kuhn, D.R.; and Cherniavsky, J.C.; Report on a Workshop on the Assurance of High Integrity Software, Proceedings 6th Annual Conference on Computer Assurance (COMPASS 91) IEEE. Computer Society Press, 1991.

Fisher, William M.; Benchmarks for the Evaluation of Speech Recognizer. Journal of Digital Signal Processing.

Favreau, J.P.; Bochmann, G.V.; Mondin-Monval, P.; Open Issues in OSI Protocol Development and Conformance Testing. Computer Networks '91, Poland.

Podio, Fernando L.; The NIST Program for Investigating Error Reporting Capabilities of Optical Disk Drives. Proceedings of Workshop on Monitoring and Reporting Techniques for Error Rate and Error Distribution in Optical Disk Systems. August 1991.

Wilson, Charles L.; Faust: A Vision-Based Neural Network Multi-Map Pattern Recognition Architecture. Proceedings of the International Joint Conference on Neural Networks ' 91 .

Fong. Elizabeth N.; Methodologies for Evaluation of Persistent Object Base Technology. Proceedings of Open Object Oriented Data Base Workshop Il. September 1991.

Sergio, Cardenas-Garcia; and Zelkowitz, Marvin V.; A Management Tool for Evaluation of Software Designs. IEEE Transactions on Software Engineering 17(9). September 1991.

Lyon, Gordon; Book Review of The Art of Computer Systems Performance Analysis by R. Jain. IEEE Design \& Test of Computers. September 1991.

Wardle, Caroline; Wallace, D.R.; Khorramshagol, Eugene; McGuire, Eugene; Kaplan, Bonnie; SQA Standards and Total Quality Management, Pacific Northwest Software Quality Conference. October 1991.

Harman, Donna; The SMART System After 30 Years - Evaluation Issues Then and Now. 14th International Conference on Research and Development in Information Retrieval. October 1991. 
Saltman, Roy G.; Standards for EDI. Second Annual Conference and Exposition on EDI for Government.

Cherniavsky, J.C.; Kuhn, D.R.; and Wallace, D.R.; High Integrity Software Standards Activities at NIST. Proceedings, Pacific Northwest Software Quality Conference. October 1991.

Saltman, Roy G.; Developing Standards for Computer Voting Systems. Computer Professionals for Social Responsibility, Washington, DC Chapter.

Newton, Judith J.; Naming Convention Verification Technical Report. ANSI Technical Report.

Schneeman, Richard D.; Using Client-Server Software Techniques in the Open System Environment to Facilitate Multimedia Application Portability.

Spielman, Frankie E.; Electronic Image Management Standards, Open Document Architecture (ODA) and Document Interchange. Handout for Document Image Management in Govt. Conference.

Wilkinson, R. Allen; Segmenting Text Images with Massively Parallel Machines. Proceedings of SPIE. October 1991.

Moline, Judi; Designing Multimedia Systems for Museum Objects and Their Documentation. ASIS Monograph.

Harman, Donna K.; Inverted Files Relevance Feedback and Other Query Modification Techniques. Information Retrieval (chapter in book).

Wilson, Charles L.: Massively Parallel Implementation of Neural Network Architectures. SPIE/SPSE Symposium on Electronic Imaging Science and Technology. Feb 24-Mar 1, 1991.

Fisher, William M.; Benchmarks for the Evaluation of Speech Recognizers. Digital Signal Processing - A Review Journal, Academic Press.

Garofolo, John S.; Escaping the UNIX Tar Pit Producing CD-ROMs in the UNIX Environment. Disk Magazine for CD/ROM Publisher.

Garris, Michael D.; Analysis of a Biologically Motivated Neural Network for Character Recognition. ANNA - 91 Proceedings.

Moline, Judi,: Designing Multimedia Systems for Museum Objects and Their Documentation. Spectra Publisher. 
Williamson, Mark P.; An Initial Appraisal of the Potential Risks Associated with the Storage of Data on 3480 Type Media and A Summary of the Care and Handling Procedures Required to Minimize these Risks. Proceedings Tape Head Interface Committee - Abstract only to be published.

Linn, Richard J.; Conformance Testing Methodologies and Architectures for OSI Protocols. IEEE Computer Society Tutorial.

Newton, Judith J.; Data Administration Management Association Symposium. NIST Journal of Research. September/October 1991.

Nightingale, J.S.; Report of the ISO/IEC Workshop on Worldwide Recognition of OSI Test Results, May 6-8, 1991, NIST Journal of Research. November/December 1991.

Radack, Shirley M.; A Report on the 6th International Conference on the Application of Standards for Open Systems. NIST Journal of Research. November/December 1991.

Kuhn, D.R.; IEEE'S POSIX: Making Progress. IEEE Spectrum. December 1991.

Gallagher, Leonard J.; Database Management Standards: Status and Applicability. Computer Standards and lnterfaces. North Holland Publishing Company. December 1991.

Cugini, John V.; Interactive Conformance Testing for PHIGS. To be published in EUROGRAPHICS '91.

Garris, Michael; Design and Collection of a Handwriting Sample Image

Database. Social Science Computing Journal Vol. 10. 1992.

Smid, Miles E; Cryptography. To be published in Handbook of lnformation Security Management.

Newton, Judith J.; Developing and Applying Data Entity Naming Conventions. To be published in Data Resource Management, Auerbach Publishers.

Harman, Donna; User Testing a Statistical Information Retrieval System. The Bulletin of the American Computing Journal, Vol. 10, 1992. 


\section{CONFERENCES AND WORKSHOPS}

October 1990 - December 1991

1990

October 1-4 13th National Computer Security Conference (cosponsored by the National Computer Security Center [NCSC])

October 2-4 6th International Conference on the Application of Standards for Open Systems (cosponsored by the Institute of Electrical and Electronics Engineers (IEEE) and the IEEE Computer Society)

October 11-12 Workshop on Integrated Software Engineering Environments (ISEE)

October 15 Workshop on Formatted Document Recognition

October 23 Lecture Series on High Integrity Systems

October 24-25 Department of Defense Electronic Data Interchange

October 29 Multimedia Systems Workshop

November 5-8 North American ISDN Users' Forum

November 15 Applications Portability Profile/Open Systems Environment (APP/OSE) Workshop

December 10-14 OSI Implementors Workshop (cosponsored by the IEEE Computer Society)

December 17 Lecture Series on High Integrity System

1991

January 17-18 International Workshop on Conformance Testing of Programming Language Standards

January 22-23 Workshop on the Assurance of High Integrity Software

February 12 Lecture Series on High Integrity Systems

February 20-21 Computer Security Educators Conference

Feb 26-Mar 1 North American ISDN Users' Forum

March 11-15 OSI Implementors Workshop (cosponsored by the IEEE Computer Society)

March 22 Lecture Series on High Integrity Systems

April 9-10 Workshop on Security Labels for Open Systems

May 2-3 Department of Defense Electronic Data Interchange 
May 6-8 Worldwide Recognition of OSI Test Results (cosponsored by the International Organization for Standardization and the International Electrotechnical Commission)

May 9 Applications Portability Profile/Open Systems Environment (APP/OSE) Workshop

May 14-15 Fourth Annual Data Administration Management Association (DAMA) Symposium (cosponsored by DAMA National Capital Region)

June 3 Lecture Series on High Integrity Systems

June 10-14 OSI Implementors Workshop (cosponsored by the IEEE Computer Society)

June 17 Workshop on X.400 in the Federal Government

June 17-20 North American ISDN Users' Forum

June 20 29th Association for Computing Machinery (ACM) Annual Technical Symposium

June 24-28 6th Annual Conference on Computer Assurance (COMPASS '91)(cosponsored by the IEEE National Capital Area Council and the IEEE Aerospace and Electronic Systems Society)

August 5 Workshop on Monitoring and Reporting Techniques for Error Rates and Error Distributions in Optical Disk Systems

August 6-8 Risk Management Workshop

September 9-13 OSI Implementors Workshop (cosponsored by the IEEE Computer Society)

September 27 Hypermedia Lecture Series

October 1-4 North American ISDN Users' Forum

October 1-4 14th National Computer Security Conference (cosponsored by NCSC)

October 11 Lecture Series on High Integrity Software

October 11 Lecture on Object-Oriented Databases

November 8 Hypermedia Lecture Series

November 8 Lecture Series on High Integrity Software

November 12 Applications Portability Profile/Open Systems Environment (APP/OSE) Workshop

November 18 Computer Security Awareness Seminar

December 2 Computer Security Day

December 3 Lecture Series on High Integrity Systems

December 9-13 OSI Implementors Workshop (cosponsored by the IEEE Computer Society) 


\section{PLANNED CONFERENCES AND WORKSHOPS}

1992

January 24 Hypermedia Lecture Series

February 14 Lecture Series on High Integrity Systems

February 18-21 Spatial Data Transfer Standard Workshop

February 25-28 North American ISDN Users' Forum

March 6 Hypermedia Lecture Series

March 9-13 OSI Implementors Workshop (cosponsored by the IEEE Computer Society)

April 3 Lecture Series on High Integrity Systems

April 10 Hypermedia Lecture Series

April 27-29 CD-ROM Technical Conference

May 18 Lecture Series on High Integrity Systems

June 2-5 North American ISDN Users' Forum

June 8-12 OSI Implementors Workshop (cosponsored by the IEEE Computer Society)

June 15-19 COMPASS '92 (cosponsored by the IEEE National Area Council and the IEEE Aerospace and Electronics Systems Society)

September 21-25 OSI Implementors Workshop (cosponsored by the IEEE Computer Society)

October 27-30 North American ISDN Users' Forum

November 16-20 Transcontinental ISDN Project '92

December 14-18 OSI Implementors Workshop (cosponsored by the IEEE Computer Society) 


\section{TALKS}

During the past year, CSL staff members presented papers and gave talks to a large number of external organizations, including the following:

ACM/NIST 29th Annual Technical Symposium

American Bar Association

American National Standards Institute (ANSI)

American Society for Industrial Security

Association for Computing Machinery (ACM)

Association for Federal Information Resources Management (AFFIRM)

Canadian Communications Security Establishment

Carnegie-Mellon University

CASE Expo, Spring 1991

COMPASS'91. Annual Conference on Computer Assurance

Computer-aided Acquisition and Logistic Support (CALS) Expo'91

Conference and Exposition

Computer Security Institute

Computer Integrated Manufacturing Conference

Computer Networks '91

Corporation for Open Systems

Data Administration Management Association (DAMA)

DARPA

Data Interchange Standards Association

Data Processing Management Association

DECUS Symposium

Defense \& Government Computer Graphics Conference

Defense Finance and Accounting Service

Department of Commerce, Bureau of the Census

Department of Defense

Department of Health and Human Services

Eastern Small College Computing Conference

EDI and Government Computer News Conference

EDP Auditors Association

Entity-Relationship User's Group

Federal ADP Users Group

Federal Computer Conference

Federation of Government Information Processing Councils

Geographic Information and Spacial Data Exposition and Conference

George Washington University

Government Technology Conference and Exposition

Graphics Users Workshop 
Hewlett-Packard

Honeywell Federal Systems Division

Information Systems Security Association (ISSA) '91

INFORUM

Institute for Supercomputing Research of Japan

Institute of Electrical and Electronics Engineers (IEEE)

Institute of Engineers, Australia Conference

Institut National de Telecommunications, Evry, France

Interagency Working Group on Management of Data for Global Change Internal Revenue Service

International Conference on Research and Development in

Information Retrieval

International Data Administration Symposium

International Joint Conference on Neural Networks '91

International Neural Network Society

INTEROP '91

Joint Data Standardization Conference

Joint International Conference on Vector and Parallel Processing,

Zurich. Switzerland

Los Alamos National Laboratory

MAP/TOP Users Group

MITRE Corporation

National Aeronautics and Space Administration

National Archives and Records Administration

National Association of State Election Directors

National Communications Forum, Chicago

National Computer Graphics Association

National Computer Security Center

National Computer Security Conference

National Contract Management Association

National Endowment for the Humanities

National Science Foundation

National Security Industrial Association

Naval Surface Warfare Center

NIST Applications Portability Profile/Open Systems Environment

(APP/OSE) Workshops

NIST Fingerprint Image Analysis Workshop

NIST OSI Implementors Workshop (OIW)

North American Integrated Services Digital Network Users' Forum

(NIU-Forum) 
Ohio Supercomputing Research Center

Open Software Foundation

Quality Assurance Institute

Second National Conference on Optical Storage Laws and Regulations

Sixth International Conference on Standards for Open Systems

Smithsonian Seminar on Scientific Imaging

Software AG Federal Industry Group

Standards for Computer Integrated Manufacturing Conference

Supercomputer Technology Conference

Symposium on High-Speed Telecommunications and Integrated Hospital Imagery

Tenth International IFIP WG6.1 Symposium on Protocol Specification, Testing, and Verification

Thirteenth International Conference on Research and Development in Information Retrieval

Unigraphics Users Group

University of Arizona

University of Maryland

UNLX International

X/OPEN 


\section{ELECTRONIC BULLETIN BOARDS}

CSL operates four electronic bulletin boards for information exchange:

Information about computer security

(301) $948-5717$

9600 baud only

(301) $948-5140$

Information about data management

(301) $948-2048$

activities and applications

and $948-2059$

Information about Open Systems

(301) 869-8630

Interconnection standards activities

Information about the North American

(301) 869-7281

Integrated Services Digital Network

(ISDN) Users' Forum (NIU-Forum)

NIST operates the following bulletin board:

Information about the Computer-aided

(301) 921-9842

Acquisition and Logistic Support (CALS)

and $948-7438$

Program

Users can reach the bulletin boards by dialing the numbers listed above.

Terminals should have the following capabilities:

ASCII, 300, 1200, or 2400 baud $(9600$ baud available for computer security bulletin board only), 8 bits with no parity or 7 bits with even parity, 1 stop bit.

If a connection is not established at the end of two rings or if the line is busy, hang up and try again.

After "CONNECT," strike the carriage return twice and the system will be accessed. The system will now guide you through the bulletin board by asking key questions and providing helpful menus. 


\section{USER GROUPS SPONSORED BY CSL}

The Open Systems Interconnection (OSI) Implementors Workshop (OIW) meets four times a year to discuss detailed implementation specifications for OSI standards.

CONTACT: $\quad$ Tim Boland

B-217 Technology Building

National Institute of Standards and Technology

Gaithersburg, MD 20899

Telephone: (301) 975-3608

The joint ISDN Users' Workshop and ISDN Implementors' Workshop of the North American ISDN Users' Forum (NIU-Forum) meets three times a year to address application requirements and to develop application profiles for ISDN products and services.

CONTACT: $\quad$ Dawn Hoffman

B-364 Materials Building

National Institute of Standards and Technology

Gaithersburg, MD 20899

Telephone: (301) 975-2937

The Applications Portability Profile/Open System Environment (APP/OSE) User Forum meets twice a year to identify federal requirements and to discuss the development of an architectural approach to applications portability in an open system environment.

CONTACT: James Hall

B-266 Technology Building

National Institute of Standards and Technology

Gaithersburg, MD 20899

Telephone: (301) 975-3273

The Graphics in Government (GIG) Users' Group focuses on the specific and unique graphics technology needs of federal agencies, increases communication among agencies, and advises government managers and users about current and planned activities to assist agencies in the area of graphics technology and standards.

CONTACT: $\quad$ Daniel Benigni

A-266 Technology Building

National Institute of Standards and Technology

Gaithersburg, MD 20899

Telephone: (301) 975-3266 


\section{FIPS PUBLICATIONS LIST BY FIPS NUMBER}

\section{December}

FIPS NO. CATEGORY TITLE-DATE

0

$1-2$

2-1

3-1

4-1

$5-2$

$6-4$

9-1

(1) P General Description of FIPS Register 68 Nov 01

(2\&3) S Code for Information Interchange, Its

Representations, Subsets, and Extensions (ANSI X3.4-1977, X3.32-1973, X3.41-1974)

84 Nov 14

(2) S Perforated Tape Code for Information Inter-

change (ANSI X3.6-1965/R1983 \& R1991)

84 Nov 14

(2) S Recorded Magnetic Tape for Information Inter-

change (800 CPI, NRZI) (ANSI X3.22-1973)

73 June 30

(4) S Representation for Calendar Date and Ordinal

Date for Information Interchange

(ANSI X3.30-1985/R1991)

88 Jan 27

(4) S Codes for the Identification of the States, the

District of Columbia and the Outlying Areas of the United States, and Associated Areas

87 May 28

(4) S Counties and Equivalent Entities of the

United States, Its Possessions, and Associated Areas

90 Aug 31

WITHDRAWN

(4) S Metropolitan Statistical Areas (MSAs) (Including

CMSAs, PMSAs, and NECMAs)

84 Oct 31

(4) S Congressional Districts of the U.S.

90 Nov 30

Category Key: (1) General Publications (2) Hardware Standards/guidelines (3) Software Standards/guidelines (4) Data Standards/guidelines (5) Computer Security Standards/guidelines (6) ADP Operations Standards/guidelines (7) Computer-Related Telecommunications Standards (8) Conformance Tests

S-Standard G-Guideline P-Program Information Document T-Conformance Tests 
FIPS NO. CATEGORY TITLE-DATE

10-3 (4) S Countries, Dependencies, Areas of Special

Sovereignty, and Their Principal Admin. Divs.

$84 \mathrm{Feb} 09$

*11-3 (3) G Guideline: American National Dictionary for lnform.

Systems (ANS1 X3.172-1990)

$91 \mathrm{Feb} 01$

$12-2$

WITHDRAWN

13 (2) S Rectangular Holes in Twelve-Row Punched Cards

(ANSI X3.21-1967/R1980 \& R1991)

71 Oct 01

$14-1$

(2) S Hollerith Punched Card Code (ANSI X3.26-1980/R1991)

80 Dec 24

15

WITHDRAWN

16-1

(7) S Bit Sequencing of Code for Information Inter-

change in Serial-By-Bit Data Transmission

(ANSI X3.15-1976/R1983 \& R1990)

77 Sept 01

$17-1$

(7) S Character Structure and Char. Parity Sense for

Serial-By-Bit Data Communication in the Code for

Inform. lnterehg. (ANS1 X3.16-1976/R1983 \& R1990)

77 Sept 01

18-1

(6) S WITHDRAWN

1

19-1

(4) G Catalog of Widely Used Code Sets

$85 \mathrm{Jan} 07$

2

20

WITHDRAWN

1

*21-3

(3) S COBOL (ANSI X3.23-1985 \& X3.23A-1989)

90 Jan 12

1

*Approved in 1991

Category Key: (1) General Publications (2) Hardware Standards/guidelines (3) Software Standards/guidelines (4) Data Standards/guidelines (5) Computer Security

Standards/guidelines (6) ADP Operations Standards/guidelines (7) Computer-Related Telecommunications Standards (8) Conformance Tests

S-Standard G-Guideline P-Program Information Document T-Conformance Tests 
22-1 (7) S Synchronous Signaling Rates Between Data

Terminal and Data Communication Equip.

(ANSI X3.1-1976)

77 Sept 01

WITHDRAWN

(2) S Recorded Magnetic Tape for Information Interchg.

(1600 CPI, Phase Encoded) (ANSI X3.39-1973)

73 June 30

(2) S One-Inch Perforated Paper Tape for Information

Interchange (ANSI X3.18-1967/R1974\&1982 \& 1990)

73 June 30

(2) S Take-Up Reels for One-Inch Perforated Tape for Information Interchg. (ANSI X3.20-1967/R1982 \& 1990)

73 June 30

(4) P Standardization of Data Elements and Representations

73 Dec 05

29-2 (1\&3) P Interpretation Procedures for Federal Information Processing Standards for Software

87 Sept 14

(3) S Software Summary for Describing Computer Programs and Automated Data Systems

74 June 30

(5) G Guidelines for Automatic Data Processing Physical Security and Risk Management

74 June-

(2) S Character Sets for Optical Char. Recognition (OCR) (ANSI X3.2-1970/R1976,X3.17-1981/R1989, X3.49-1975/ $\mathrm{R} 1982$ \& 1989)

82 June 25

(2) S Character Set for Handprinting (ANSI X3.45-1982/R1989) 84 Nov 05

Category Key: (1) General Publications (2) Hardware Standards/guidelines (3) Software Standards/guidelines (4) Data Standards/guidelines (5) Computer Security Standards/guidelines (6) ADP Operations Standards/guidelines (7) Computer-Related Telecommunications Standards (8) Conformance Tests

S-Standard G-Guideline P-Program Information Document T-Conformance Tests 
34 (1) P Guide for the Use of International System of Units (SI) in Federal Information Processing Standards Publications 75 Jan 01

35

36

37

38

39

40

$42-1$

43

46- 1
WITHDRAWN

WITHDRAWN

WITHDRAWN

(3) G Guidelines for Documentation of Computer Programs and Automated Data Systems

$76 \mathrm{Feb} 15$

(5) G Glossary for Computer Systems Security

76 Feb 15

(2) G Guideline for Optical Character Recognition Forms

76 May 01

(5) G Computer Security Guidelines for Implementing the

Privacy Act of 1974

75 May 30

(6) G Guidelines for Benchmarking ADP Systems in the

Competitive Procurement Environment

77 May 15

WITHDRAWN

WITHDRAWN

(4) G Guide for the Development, Implementation \& Maintenance of Standards for the Representation of Computer Processed Data Elements

76 Sept 30

(5) S Data Encryption Standard

88 Jan 22

WITHDRAWN

(5) G Guidelines on Evaluation of Techniques for Automated Personal Identification

77 Apr 01

Category Key: (1) General Publications (2) Hardware Standards/guidelines (3) Software Standards/guidelines (4) Data Standards/guidelines (5) Computer Security Standards/guidelines (6) ADP Operations Standards/guidelines (7) Computer-Related Telecommunications Standards (8) Conformance Tests

S-Standard G-Guideline P-Program Information Document T-Conformance Tests 
(6) G Guideline on Computer Performance Management:

An Introduction

77 May 01

(2) S Recorded Magnetic Tape for Information Interchange, 6250 cpi (246 cpmm), Group Coded Recording (ANSI X3.54-1976)

$78 \mathrm{Feb} 01$

(2) S Magnetic Tape Cassettes for Information Interchange (3.810 $\mathrm{mm}[0.150 \mathrm{in}$ ] Tape at $32 \mathrm{bpmm}$ [800bpi],PE) (ANSI X3.48-1977)

$78 \mathrm{Feb} 01$

(2) S Recorded Magnetic Tape Cartridge for Inform. Interchange., 4-Track, $6.30 \mathrm{~mm}$ (1/4in), 63 bpmm (1600 bpi), Phase Encoded (ANSI X3.56-1977) 78 July 15

(3) S Transmittal Form for Describing Computer Magnetic Tape File Properties 78 Apr 01

(2) S Computer Output Microform (COM) Formats and

Reduction Ratios, 16mm and 105mm (ANSI/AIIM MS5-1991

\& MS14-1988)

91 Jan 15

(4) G Guideline: Codes for Named Populated Places Primary County Divisions, and Other Locational Entities of the United States and Outlying Areas

87 Jan 16

(4) G Same as 55DC except without codes $87 \mathrm{Feb} 03$

(6) G Guideline for Managing Multivendor Plug-Compatible ADP Systems

78 Sept 15

(6) G Guidelines for the Measurement of Interactive Computer Service Response Time and Turnaround Time 78 Aug 01

\footnotetext{
*Approved in 1991

Category Key: (1) General Publications (2) Hardware Standards/guidelines (3) Software Standards/guidelines (4) Data Standards/guidelines (5) Computer Security Standards/guidelines (6) ADP Operations Standards/guidelines (7) Computer-Related Telecommunications Standards (8) Conformance Tests

S-Standard G-Guideline P-Program Information Document T-Conformance Tests
} 
FIPS NO. CATEGORY TITLE-DATE

58-1 (4) S Representations of Local Time of the Day for Information Interchange (ANSI X3.43-1986) 88 Jan 27

59

$60-2$

$61-1$

62

63-1

(4) S Representations of Universal Time, Local Time

Differentials, and United States Time Zone

References for Information Interchange

(ANSI X3.51-1975)

$79 \mathrm{Feb} 01$

(2) S I/O Channel Interface

83 July 29

(2) S ChanneI Level Power Control Interface 82 July 13

(2) S Operational Specification for Magnetic Tape

Subsystems

79 Feb 16 2+F.R. notice

(2) S Operational Specification for Variable Block

Rotating Mass Storage Subsystems

83 Apr 14

63-1 SUPPLEMENT Additional Operational Specs for VBRMSS

83 Apr 14

1

64 (3) G Guidelines for Documentation of Computer Programs and Automated Data Systems for the Initiation Phase 79 Aug 01

(5) G Guideline for Automatic Data Processing Risk Analysis 79 Aug 01

66

(4) S Standard Industrial Classification (SIC) Codes

79 Aug 15

67

(2) G Guideline for Selection of Data Entry Equipment

79 Sept 30

$68-2$

(3) S BASIC (ANSI X3.113-1987)

87 Aug 28

69-1 (3) $\mathrm{S}$ FORTRAN (ANSI X3.9-1978/R1989)

85 Dec 24

Category Key: (1) General Publications (2) Hardware Standards/guidelines (3) Software Standards/guidelines (4) Data Standards/guidelines (5) Computer Security Standards/guidelines (6) ADP Operations Standards/guidelines (7) Computer-Related Telecommunications Standards (8) Conformance Tests

S-Standard G-Guideline P-Program Information Document T-Conformance Tests 
$70-1$

(4) S Representation of Geographic Point Locations for Information Interchange (ANSI X3.61-1986)

86 Nov 14

(7) S Advanced Data Communication Control Procedures

(ADCCP) (ANSI X3.66-1979/R1990)

80 May 14

(6) G Guidelines for Measurement of Remote Batch

Computer Service

80 May 01

(5) G Guidelines for Security of Computer Applications

80 June 30

(5) G Guidelines for Implementing and Using the NBS Data

Encryption Standard

81 Apr 01

(6) G Guideline on Constructing Benchmarks for ADP System

Acquisitions

80 Sept 18

(3) G Guideline for Planning and Using a Data Dictionary System

80 Aug 20

(3) G Guideline for Planning and Management of Database Applications 80 Sept 01

(7) G Guideline for Implementing Advanced Data Communication

Control Procs (ADCCP)

80 Sept 26

(3) S Magnetic Tape Labels and File Structure for Information

Interchange (ANSI X3.27-1978)

80 Oct 17

WITHDRAWN

1

(5) S DES Modes of Operation

80 Dec 02

(2) G Guideline for Inspection and Quality Control for Alphanumeric Computer-Output Microforms (AIIM (NMA) MS1 - 1980)

80 Sept 26

Category Key: (1) General Publications (2) Hardware Standards/guidelines (3) Software Standards/guidelines (4) Data Standards/guidelines (5) Computer Security Standards/guidelines (6) ADP Operations Standards/guidelines (7) Computer-Related Telecommunications Standards (8) Conformance Tests

S-Standard G-Guideline P-Program Information Document T-Conformance Tests 
83 (5) G Guideline on User Authentication Techniques for Computer Network Access Control 80 Sept 29

(2) S Microfilm Readers (ANSI/AIIM(NMA) MS20-1979) 80 Oct 31

(2) S Optical Character Recognition (OCR) Inks (ANSI X3.86-1980/R1987) 80 Nov 07

(2) S Additional Controls for Use with Amer. Natl. Std. Code for Inform. Interchg. (ANSI X3.64-1979/R1990) 81 Jan 29

(5) G Guidelines for ADP Contingency Planning 81 Mar 27

(3) G Guideline on Intregity Assurance and Control in Database Administration 81 Aug 14

(2) S Optical Character Recognition (OCR) Character Positioning (ANSI X3.93M-1981/R1989) 81 Sept 04

(2) G Guideline for Optical Character Recognition (OCR) Print Quality (ANSI X3.99-1983/R1991) 83 Sept 29

(4) G Guideline for Standard Occupational Classification (SOC) Codes $83 \mathrm{Feb} 24$

(2) S Parallel Recorded Magnetic Tape Cartridge for InformationI nterchange, 4-Track, $6.30 \mathrm{~mm}$ (1/4 in), 63 bpmm (1600 bpi), Phase Encoded (ANSI X3.72-1981/R1987) 82 June 29

(2) G Guideline on Electrical Power for ADP Installations 83 Sept 21

Category Key: (1) General Publications (2) Hardware Standards/guidelines (3) Software Standards/guidelines (4) Data Standards/guidelines (5) Computer Security Standards/guidelines (6) ADP Operations Standards/guidelines (7) Computer-Related Telecommunications Standards (8) Conformance Tests S-Standard G-Guideline P-Program Information Document $\boldsymbol{T}$-Conformance Tests 
95 (4) S Codes for the Identification of Federal and Federally-

Assisted Organizations

82 Dec 23

(6) G Guideline for Developing and Implementing a Charging System for Data Processing Services 82 Dec 06

(2) S Operational Specifications for Fixed Block Rotating Mass Storage Subsystems

$83 \mathrm{Feb} 04$

WITHDRAWN

(3) G Guideline: A Framework for the Evaluation and

Comparison of Software Development Tools

83 Mar 31

(7) S Interface Between Data Terminal Equipment (DTE) and Data Circuit-Terminating Equipment (DCE) for Operation with Packet-Switched Data Networks (PSDN), or Between Two DTEs, by Dedicated Circuit (ANSI X3.100-1989)

91 Mar 20

(3) G Guideline for Lifecycle Validation, Verification, and Testing of Computer Software 83 June 06

(5) G Guideline for Computer Security Certification and Accreditation

83 Sept 27

(4) S Codes for the Identification of Hydrologic Units in the United States and the Caribbean Outlying Areas (USGS/CIRCULAR \#878-A\& ANSI X3.145-1986) 83 Nov 15

(4) S ANS Codes for the Representation of Names of Countries, Dependencies, and Areas of Special Sovereignty for Information Interchange 86 May 12

\footnotetext{
*Approved in 1991

Category Key: (1) General Publications (2) Hardware Standards/guidelines (3) Software Standards/guidelines (4) Data Standards/guidelines (5) Computer Security Standards/guidelines (6) ADP Operations Standards/guidelines (7) Computer-Related Telecommunications Standards (8) Conformance Tests

S-Standard G-Guideline P-Program Information Document T-Conformance Tests
} 
105 (3) G Guideline for Software Documentation Management 84 June 06

(3) G Guideline on Software Maintenance 84 June 15

(2\&3) S Local Area Netwurks: Baseband Carrier Sense Multiple Access with Collision Detection Access Method and Physical Layer Specifications and Link Layer Protocol (ANSI/IEEE 802.2\&802.3) 84 Oct 31

(2) S Alphanumeric Computer Output Microform Quality Test Slide (AIIM MS28-1983) 84 Nov 05

(3) S Pascal (ANSI/IEEE 770X3.97-1983/R1990) 85 Jan 16

(3) G Guideline for Choosing a Data Management Approach 84 Dec 11

(2) S Storage Module Interfaces (w/extens. for enhanced storage module interfaces) (ANSI X3.91M-1982) 85 Apr 18

(5) S Password Usage

85 May 30

(5) S Computer Data Authentication 85 May 30

(2) S $200 \mathrm{~mm}$ (8in) Flexible Disk Cartridge Track Format Using Two-Frequency Modulation Recording at 6631 bprad on One Side - 1.9 tpmm (48 tpi) for Information Interchange (ISO $5654 / 2$ ) 85 Sept 30

(2) $\mathrm{S} 200 \mathrm{~mm}$ (8in) Flexible Disk Cartridge Track Format Using Modified Frequency Modulation Recording at 13262 bprad on Two-Sides - 1.9 tpmm (48 tpi) for Information Interchange (ISO 7065/2) 85 Sept 30

Category Key: (1) General Publications (2) Hardware Standards/guidelines (3) Software Standards/guidelines (4) Data Standards/guidelines (5) Computer Security Standards/guidelines (6) ADP Operations Standards/guidelines (7) Computer-Related Telecommunications Standards (8) Conformance Tests

S-Standard G-Guideline P-Program Information Document T-Conformance Tests 
116 (2) $\mathrm{S} 130 \mathrm{~mm}$ (5.25 in) Flexible Disk Cartridge Track Format

Using Two-Frequency Recording at 3979 bprad on One Side

- 1.9 tpmm (48 tpi) for InformationI nterchange (ISO 6596/2)

85 Sept 30

(2) S $130 \mathrm{~mm}$ (5.25 in) Flexible Disk Cartridge Track Format

Using Modified Frequency Modulation Recording at

7958 bprad on two sides -1.9 tpmm (48 tpi) for

Information interchange (ISO 7487/3)

85 Sept 30

118 (3) S Flexible Disk Cartridge Labelling and File Structure

for Information Interchange (ISO 7665)

85 Sept 30

(3) S Ada (ANSI/MIL-STD-1815A-1983)

85 Nov 08

*120-1 (3) S Graphical Kernel System (GKS) (ANSI X3.124-1985,

X3.124.1-1985, X3.124.2-1988, X3.124.3-1989)

91 Jan 08

(2\&3) S Videotex/Teletext Presentation Level Protocol Syntax

(North American PLPS) (ANSI X3.110-1983(R1991)/

CS T500-1983)

86 May 06

(8) $\mathrm{T}$ Conformance Tests for FIPS PUB 100 Version of CCITT 1980 Recommendation X.25, etc.

86 May 28

(3) S Specification for a Data Descriptive File for Information Interchange (DDF) (ANSI/ISO 821 1-1985)

86 Sept 19

(3) G Guideline on Functional Specifications for Database

Management Systems

86 Sept 30

(3) S MUMPS (ANSI/MDC X11.1-1984)

86 Nov 4

(3) S Database Language NDL (ANSI X3.133-1986)

87 Mar 10

*Approved in 1991

Category Key: (1) General Publications (2) Hardware Standards/guidelines (3) Software Standards/guidelines (4) Data Standards/guidelines (5) Computer Security Standards/guidelines (6) ADP Operations Standards/guidelines (7) Computer-Related Telecommunications Standards (8) Conformance Tests

S-Standard G-Guideline P-Program Information Document $\mathbf{T}$-Conformance Tests 
127-1 (3) S Database Language SQL

(ANSI X3.135-1989 \& X3.168-1989)

$90 \mathrm{Feb} 02$

(3) S Computer Graphics Metafile (CGM) (ANSI X3.122-1986)

87 Mar 16

(2) S Optical Character Recognition (OCR) - Dot Matrix

Character Sets for OCR-MA (ANSI X3.111-1986)

87 May 06

(2) S Intelligent Peripheral Interface (IPI) (ANSI X3.129-1986,

X3.130-1986, X3.132-1987, and X3.147-1987)

87 July 16

(2) S Small Computer System Interface (SCSI) (ANSI X3.131-1986)

87 July 16

(3) G Guideline for Software Verification and Validation

Plans (ANSI/IEEE 1012-1986)

87 Nov 19

(7) S Coding and Modulation Requirements for 2,400 Bit/

Second Modems

86 June 02

(7) S Coding and Modulation Requirements for 4800 Bit/

Second Modems

88 Nov 04

(7) S Coding and Modulation Requirements for Duplex 9600

Bit/Second Modems

81 March

(7) S Coding and Modulation Requirements for Duplex 600 and $1200 \mathrm{Bit} /$ Second Modems

80 June 16

(7) S Analog to Digital Conversion of Voice by 2,400 Bit/

Second Linear Predictive Coding

84 Nov 28

(7) S Electrical Characteristics of Balanced Voltage Digital Interface Circuits

75 Sept 24

Category Key: (1) General Publications (2) Hardware Standards/guidelines (3) Software Standards/guidelines (4) Data Standards/guidelines (5) Computer Security Standards/guidelines (6) ADP Operations Standards/guidelines (7) Computer-Related Telecommunications Standards (8) Conformance Tests

S-Standard G-Guideline P-Program Information Document T-Conformance Tests 

FIPS NO. CATEGORY TITLE-DATE
139 (7) S Interoperability and Security Requirements for Use of the Data Encryption Standard in the Physical Layer of Data Communications 83 Aug 3
140 (7) S General Security Requirements for Equipment Using the Data Encryption Standard 82 Apr 14

141 (7) S Interoperability and Security Requirements for Use of the Data Encryption Standard with CCITT

Group 3 Facsimile Equipment 85 Apr 04

142 (7) S Electrical Characteristics of Unbalanced Voltage Digital Interface Circuits

80 Jan 31

(7) S General Purpose 37-Position 9-Position Interface Between Data Terminal Equipment and Data Circuit-Terminating Equipment (EIA-RS-449) 85 June 10

(7) S Data Communication Systems and Services UserOriented Performance Parameters (ANSI X3.102-1983/R1990) 85 May 28

\section{WITHDRAWN}

(2\&3) S Government Open Systems Interconnection Profile (GOSIP) 91 Apr 03

(7) S Group 3 Facsimile Apparatus for Document Transmission 81 Aug 19

(7) S Procedures for Document Facsimile Transmission (EIA-RS-466) 82 Apr 14

(7) S General Aspects of Group 4 Facsimile Apparatus (EIA-536- 1988) 88 Nov 04

(7) S Facsimile Coding Schemes and Coding Control Functions for Group 4 Facsimile Apparatus (EIA-538-1988) 88 Nov 04

\footnotetext{
*Approved in 1991

Category Key: (1) General Publications (2) Hardware Standards/guidelines (3) Software Standards/guidelines (4) Data Standards/guidelines (5) Computer Security Standards/guidelines (6) ADP Operations Standards/guidelines (7) Computer-Related Telecommunications Standards (8) Conformance Tests

S-Standard G-Guideline P-Program Information Document T-Conformance Tests
} 
151-1 $\quad$ (3) S POSIX: Portable Operating System Interface for Computer Environments (IEEE 1003.1-1988)

90 Mar 28

(3) S Standard Generalized Markup Language (SGML) (ISO 8879-1986) 88 Sept 26

(3) S Programmer's Hierarchical Interactive Graphics SysteM (PHIGS) (ANSI/ISO 9592.1-3:1989, ANSI/ISO 9593.3:1990, ISO/IEC 9593.1:1990) 88 Oct 14

(7) S High Speed 25-Position Interface for Data Terminal Equipment and Data Circuit-Terminating Equipment (EIA-530-1987) 88 Nov 04

(7) S Data Communication Systems and Services User-Oriented Performance Measurement Methods (ANSI X3.141-1987) 88 Nov 04

(3) S Information Resources Dictionary System (IRDS) (ANSI X3.138-1988) 89 Apr 05

(2) G Guideline for Quality Control of Image Scanners 89 Sept 13 (ANSI/AIIM MS44-1988)

(3) S The User Interface Component of the Applications Portability Profile (MIT X Version 11, Release 3) 90 May 29

(7) S Detail Specification for 62.5- $\mu \mathrm{M}$ Core Diameter/125- $\mu \mathrm{M}$ Cladding Diameter Class Ia Multimode, Graded-Index Optical Waveguide Fibers (ANSI/EIA/TIA-492AAAA-1989)

90 Dec 27

(3) S C (ANSI X3.159-1989) 91 Mar 29 Standards/guidelines (4) Data Standards/guidelines (5) Computer Security Standards/guidelines (6) ADP Operations Standards/guidelines (7) Computer-Related Telecommunications Standards (8) Conformance Tests

S-Standard G-Guideline P-Program Information Document T-Conformance Tests 
Superintendent of Documents Publications and Subscriptions Order Form

Order Processing Code

*6191

Charge your order.

It's Easy!

To fax your orders (21)2) 512-2251)

Publications

Please Type or Print (Form is aligned for typewriter use.)

\begin{tabular}{|l|l|l|l|l|l|}
\hline Qty. & Stock Number & & $\begin{array}{c}\text { Title } \\
\text { Each }\end{array}$ & $\begin{array}{l}\text { Total } \\
\text { Price }\end{array}$ \\
\hline & & & & \\
\hline & & & & & \\
\hline & & & & & \\
\hline & & & & \\
\hline
\end{tabular}

\section{Subscriptions}

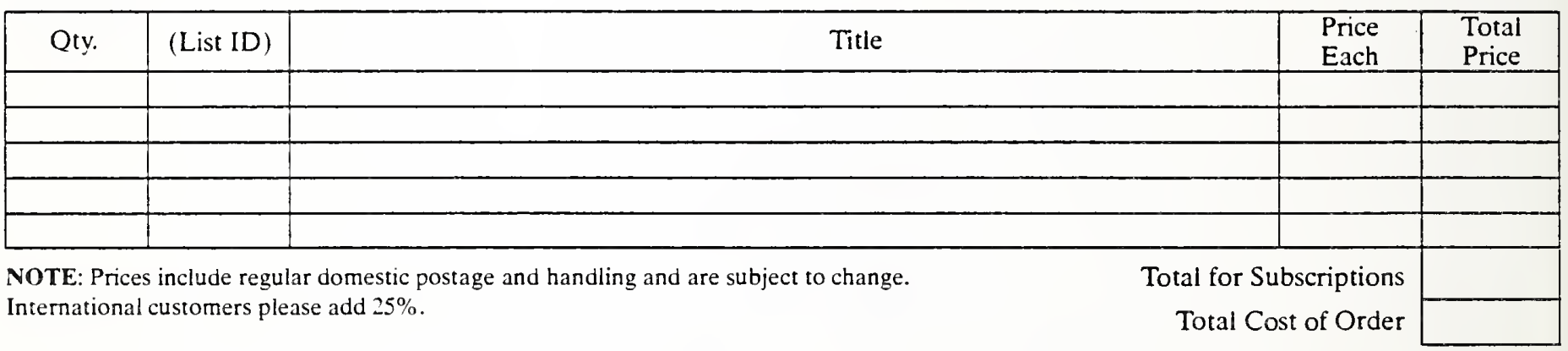

(Company or Personal Name)

(Please type or print)

(Additional address/attention line)

(Street address)

(City. State. ZIP Code)

(Daytime phone including area code)

(Purchase Order No.)

May we make your name/address available to other mailers?

\section{Please Choose Method of Payment:}

Check Payable to the Superintendent of Documents

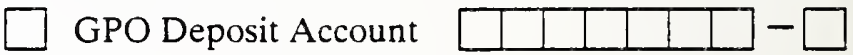

$\square$ VISA or MasterCard Account

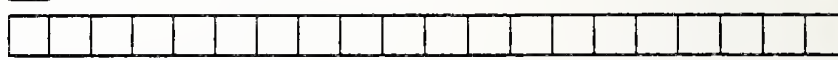

(Credit card expiration date)

Thank you for your order!

(Authorizing Signature)

$1 / 92$

Mail To: New Orders. Superintendent of Documents

P.O. Box 371954, Pittsburgh. PA 15250-7954 


\section{TELEPHONE ORDERS} Call (703) 487-4650

TELEX 89-9405 Tetecopier (703) 321-8547 Subscompoons: (703) $487-4630$

(See reverse side for RUSH and EXPRESS ordering options)

- HANDLING FEE: A handling tee is required for each order except for Express, Rush, Subscription, QuikORDER, or Pickup orders.

- SHIPPING: U.S.: Printed reports and microfiche copies are shipped Firsi Class Mail or equivalent.

FOREIGN: Regular sorvice: Printed reports and microfiche copies are shippod surface mail. Air Mail servico to Canada and Mexico: add \$3 per printed report; 75\& per microtiche copy. Air Mail service to all other addresses: add $\$ 6$ per printed report; $75 \mathrm{e}$ per microfiche copy. SUBSCRIPTIONS and standing orders are sent surface mail; contact NTIS for air mail rafos.

\section{Address Information}

PURCHASER: DATE:

\begin{tabular}{l}
\hline Last Namo \\
\hline Tive
\end{tabular}

DTic Uewre Code:

Contract No.

SHIP TO (Entor ONLY if different from purchaser):

\begin{tabular}{|c|c|}
\hline Last Nams & Finet Irutal \\
\hline Tride & \\
\hline Comperyiorgenaaton & \\
\hline
\end{tabular}

GirststeZIP

Antenion

Telephone number

\section{CinstaterzP}

Atrmion

Telephone number

\section{Method of Payment}

Charge my NTIS Deposit Account

Charge my

Acount to.

Amer. Express

$\square$ VISA $\square$ MasterCard Es.

Signature:

CheckMoney order enclosed for 5

Please bill ADD $\$ 7.50$ per order (See below for restrictions) $†$

Puches order the

\begin{tabular}{|c|c|c|c|c|c|c|}
\hline \multicolumn{2}{|l|}{3 Order Selection (For computer products, see roveres) } & \multicolumn{2}{|c|}{ QUANTITY } & \multirow[b]{2}{*}{$\begin{array}{l}\text { UNIT } \\
\text { PRICE }\end{array}$} & \multirow[b]{2}{*}{$\begin{array}{l}\text { Foroign } \\
\text { Air Mail } \\
\end{array}$} & \multirow[b]{2}{*}{$\begin{array}{l}\text { TOTAL } \\
\text { PRICE }\end{array}$} \\
\hline \begin{tabular}{l|} 
Enter NTIS order number(s) \\
(Ordening by tite only will deley your order) \\
\end{tabular} & 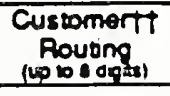 & $\begin{array}{l}\text { Prinwod } \\
\text { Copy }\end{array}$ & $\begin{array}{l}\text { Nicro- } \\
\text { fictio }\end{array}$ & & & \\
\hline \multicolumn{7}{|l|}{1.} \\
\hline \multicolumn{7}{|l|}{2.} \\
\hline \multicolumn{7}{|l|}{3.} \\
\hline \multicolumn{7}{|l|}{4.} \\
\hline \multicolumn{7}{|l|}{5.} \\
\hline \multicolumn{7}{|l|}{6.} \\
\hline \multicolumn{7}{|l|}{7.} \\
\hline \multicolumn{7}{|l|}{$\square$ OVER - Order continued on reverse } \\
\hline \multirow{2}{*}{\multicolumn{2}{|c|}{ 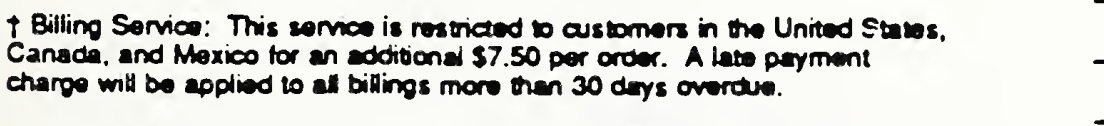 }} & \multicolumn{4}{|c|}{ 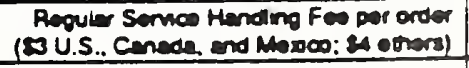 } & \\
\hline & & \multicolumn{4}{|c|}{ Bilng foo il reounnod (87.50) } & \\
\hline \multicolumn{2}{|l|}{$\begin{array}{l}\text { It Customer Routing Code: NTIS can label cech item for routing within your } \\
\text { organzabon. If you want this semvice, put your rousng code in thes box. }\end{array}$} & \multicolumn{4}{|c|}{ GRAND TOTAL } & \\
\hline
\end{tabular}




\section{Order Selection (Cont.)}

\begin{tabular}{|c|c|c|c|c|}
\hline 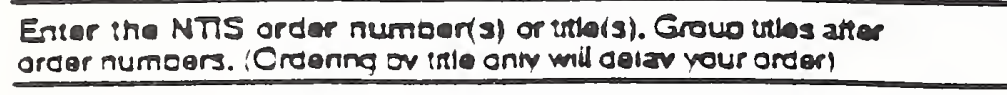 & \begin{tabular}{|l|l|} 
Custemer & P zoor \\
Routing in & Coor \\
\end{tabular} & $\begin{array}{l}\text { Micol } \\
\text { tiene }\end{array}$ & $\begin{array}{l}\text { UNT } \mid \text { Fornegn } \\
\text { PAICE! Air Maul } \\
\end{array}$ & $\begin{array}{l}\text { TCTAL } \\
\text { PAICE }\end{array}$ \\
\hline $138 \%$ & 1 & & 1 & \\
\hline /BAI & 1 & 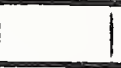 & & \\
\hline /3AI & 1 & & 1 & \\
\hline $13 A=$ & 1 & & 1 & \\
\hline$/ B A=$ & 1 & & 1 & \\
\hline /BAI & 1 & & 1 & \\
\hline $13 A 2$ & 1 & 1 & 1 & \\
\hline $13 A$ & 1 & & 1 & \\
\hline $13 A=$ & 1 & 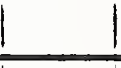 & 1 & \\
\hline 13A: & 1 & $\mid$ & 1 & \\
\hline 1BA: & 1 & & 1 & \\
\hline \multirow[t]{2}{*}{$19 A^{\circ}$} & 1 & & 1 & \\
\hline & & & Suttorai & \\
\hline
\end{tabular}

\section{ENTE. this amount on the other side of this form.}

\section{Computer Produc:s Orcer Selection}

"You have questoons abour a parteviar computer produc slense call our Camsurer Produces Suppers Group a (703) $497 d 723$.

Enter the NTIS order number(s) or wers) of the diskece or magnave :ase orodues. (Croeng oy twe ony wil doiry your order)

20.

29.

22.

22.

Al magnelk :aoos are sent ar maul or squvalent servica io bots U.S. and forengs agdresses.

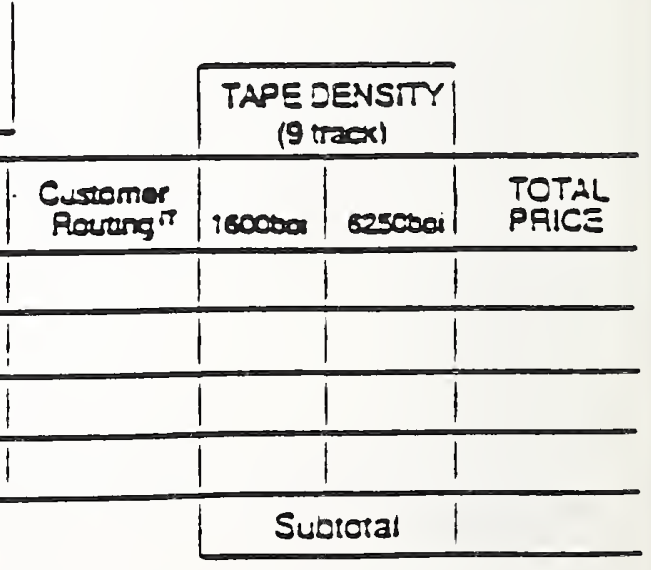

ENTE. inis amount on the other side of this form.
SPEE:AL RUSH and EXPRESS ORCE.AING OPTICNS

Teleonone: $\quad(800)$ 356-1700 in Virginia call (702) $+87-i 00$
RUSH SEAVICE: Oreers ase procassed whinn 24 nours and sem Air Mall or cqunalem.

- Pior uo as NTIS - 57.50 portion

- Dervary 10 U.S. acerossos - 510 par rem

EXPRESS SEAVICE (U.S. ACCresses Cniy): Orcers are orocassed within 23

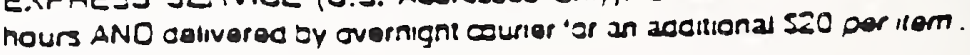




\section{BIBLIOGRAPHIC DATA SHEET}

4. TITLE AND SUBTITLE

Computer Systems Laboratory Annual Report - 1991

5. AUTHOR(S)

Elizabeth Lennon, Shirley Radack, and Ramona Roach

6. PERFORMINO ORGANIZATION (IF JOINT OR OTHER THAN NIST, SEE INSTRUCTIONS)

U.S. DEPARTMENT OF COMMERCE

NATIONAL INSTITUTE OF STANDARDS AND TECHNOLOGY

GAITHERSBURQ, MD 20899

7. CONTRACT/GRANT NUMBER

8. TYPE OF REPORT AND PERIOD COVERED

9. SPONSORING ORGAMIZATION MAME AMD COMPLETE ADDRESS (STREET, CITY, STATE, ZIP)

10. SUPPLEMENTARY NOTES

11. ABSTRACT (A 200-WORD OR LESS FACTUAL SUMMARY OF MOST SIGNIFICANT INFORMATION. IF DOCUMENT INCLUDES A SIGNIFICANT BIBLOGRAPHY OR LTERATURE SURVEY, MENTION IT HERE.)

The Computer Systems Laboratory Annual Report - 1991 describes the annual computer and related telecommunications activities and accomplishments of the Laboratory. Following the Director's Foreword, an overview of the Laboratory is presented, including a current CSL Organization Chart and selected staff accomplishments. Overviews of CSL'S five technical divisions are featured next, followed by a section on Technology Transfer which details the vehicles CSL uses to disseminate research and information to the public and technical communities. A list of Federal Information Processing Standards (FIPS) and FIPS order information conclude the annual report.

12. KEY WORDS (6 TO 12 ENTRIES; ALPHABETICAL ORDER; CAPITALZE ONLY PROPER MAMES; AND SEPARATE KEY WORDS BY SEMICOLONS) annual report; Computer systems Laboratory (CSL); computers; computer standards; Federal Information Processing Standards (FIPS); telecommunications; telecommunication standards. 


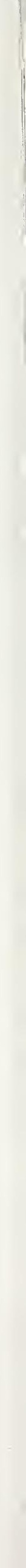



